\title{
HIGHER TORSION IN $H$-SPACES
}

\author{
BY \\ WILLIAM BROWDER(1)
}

This paper is the last of a series of papers investigating the homological structure of $H$-spaces (see $[7 ; 8 ; 9 ; 11]$ ). Here we investigate the consequences of the existence of torsion elements of higher order, that is, elements whose order is divisible by higher powers of $p$. Again, we investigate the Bockstein spectral sequence $B_{r}$ and prove certain "implication" theorems in it. The techniques of proof are more general than in [7] and [8], and we prove theorems in a more algebraic, abstract context. Thus in $\S 1$, we introduce the concept of an $H$-complex, which one may think of as an abstraction of the chain complex of an $H$-space. "Implication" theorems for the Bockstein spectral sequences of such $H$-complexes are proved in $\S \S 2$ and 3. The main results of $\S 3$ are Theorems 3.3, 3.11 and 3.12.

These theorems are then applied to $H$-spaces in $\$ 4$ and yield the following facts about (homologically) finite dimensional $H$-spaces:

(1) If some Bockstein $\beta_{r} \equiv 0$ in $B_{r}$, then $B_{r}=B_{\infty}$; i.e., $\beta_{j}=0$ for all $j>r$.

(2) If $H^{*}\left(X ; Z_{p}\right)$ is primitively generated, then all $p$-torsion is of order exactly $p$, i.e., there are no elements of order $p^{r}, r>1$, in $H^{*}(X ; Z),\left(B_{2}=B_{\infty}\right)$.

These are proved in $\$ 4$ (Corollary 4.6 and Theorem 4.9).

$\S \S 5,6$ and 7 are devoted to proving the results announced in [10] about fibering of spheres and $H$-spaces which are rational homology spheres. The implication theorems of $\$ 4$ and the results of [9] are applied to get the results:

THEOREM 5.1 $\left({ }^{2}\right)$. If $p: S^{n} \rightarrow B$ is a fibre map, with base $B$ and fibre $F$ connected polyhedra, $B \neq$ point, then $F \cong S^{1}, S^{3}$, or $S^{7}$.

THEOREM 5.2. If $X$ is a connected finite dimensional polyhedral H-space which is a rational homology sphere, then $X$ is homotopy equivalent to one of $S^{1}, S^{3}, S^{7}, P^{3}$ or $P^{7}$.

Theorem 5.1 continues the work of Borel [4] and Spanier and Whitehead [19] on this problem. If one asks in Theorem 5.1 for information on the homotopy type of $B$, one may show easily in case $F \cong S^{1}$ that $B \cong C P^{n}$ for some $n$, and if $F \cong S^{7}$, then $B \cong S^{8}$. For $S^{1}$ being $K(Z, 1)$ makes $B$ a $K(Z, 2)$ in the dimensions

Received by the editors August 3, 1962.

(1) Research partially supported by a National Science Foundation grant NSF G-15984.

(2) By polyhedron we mean $C W$ complex of finite type. By fibre map we mean fibre map in the sense of Serre, i.e., the covering homotopy theorem holds for maps of compact polyhedra. " $X \cong Y$ " means " $X$ is homotopy equivalent to $Y$." 
of its nonzero homology, which determines its homotopy type. In case $F \cong S^{7}$, then $H^{*}(B)=Z\left[y_{8}\right] /\left(y_{8}^{l}\right)$. Then it follows that $l=2$ by an argument due to Adem [3] using Steenrod operations $\bmod 3$, so that $B$ is a homology 8-sphere, simply connected, and hence a homotopy 8-sphere.

In case $F \cong S^{3}$, however, examples are known (see for instance Eells and Kuiper [13]) which show that the homotopy type is not determined by knowing $\pi_{1}(B)=0$ and $H^{*}(B)=Z\left[y_{4}\right] /\left(y_{4}^{l}\right)$. The question of the homotopy type of $B$ in this case is open.

One may define the rank of a finite dimensional $H$-space as the number of ring generators of its rational cohomology. Thus Theorem 5.2 classifies, up to homotopy type, $H$-spaces of rank 1 . It would be interesting to classify $H$-spaces of higher rank, up to homotopy type.

In $\S 8$, we give a summary of the main "implication" theorems of this paper and $[7 ; 8 ; 11]$.

We refer to [7] for a thorough discussion of the Bockstein spectral sequence.

I am indebted to E. Spanier for suggesting the problem of fibering spheres to me.

1. H-complexes and the Bockstein spectral sequence. We will consider chain complexes $C$, i.e., differential graded modules, and we will always assume that $C$ is torsion free over a principal ideal domain $R, C_{i}=0$ for $i<0$. If $f$ and $g$ are chain maps, " $f \sim g$ " means $f$ is chain homotopic to $g$.

Definition. An $H$-complex is a chain complex $C$ with chain maps $\psi: C \rightarrow C \otimes C$, $\phi: C \otimes C \rightarrow C, \eta: R \rightarrow C, \varepsilon: C \rightarrow R$, such that

(1) $\varepsilon \eta=1: R \rightarrow R$,

(2) $\eta$ is a unit for $\phi, \varepsilon$ a co-unit of $\psi$, up to homotopy, i.e., $\phi \circ(1 \otimes \eta) \sim \phi \circ(\eta \otimes 1) \sim$ identity map of $C$, where $R \otimes C$ is identified with $C$, $(\varepsilon \otimes 1) \circ \psi \sim(1 \otimes \varepsilon) \circ \psi \sim$ identity map of $C$,

(3) $\psi$ is a map of algebras up to homotopy, i.e., if $T: C \otimes C \rightarrow C \otimes C$ is defined by $T(a \otimes b)=(-1)^{p q} b \otimes a, a \in C_{p}, b \in C_{q}$, then

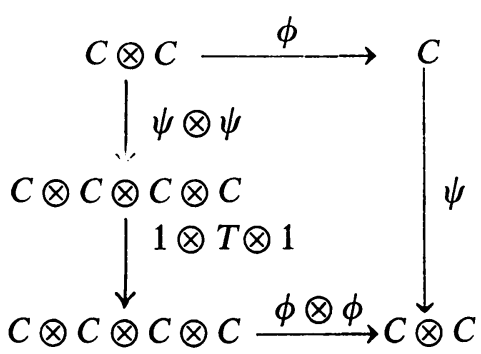

commutes up to homotopy.

Roughly speaking, an $H$-complex may be described as a "differential Hopf algebra up to homotopy." The chain and cochain groups of an $H$-space are examples of $H$-complexes over the integers. 
A chain complex (or graded module) $C$ is of finite type if $C_{i}$ is a finitely generated $R$-module for each $i$. Two chain complexes $C, C^{\prime}$ are homotopy equivalent if there are chain maps $f: C \rightarrow C^{\prime}, g: C^{\prime} \rightarrow C$, with $f \circ g \sim 1, g \circ f \sim 1 . C, C^{\prime}$ are said to have the same homotopy type, $C \sim C^{\prime}$.

Lemma 1.1. Let $C$ be a chain complex free over $R$, with $H(C)$ of finite type. Then $C$ is the homotopy type of a chain complex of finite type.

This is a standard type of chain complex argument (see $[14 ; 7]$ ).

Lemma 1.2. If $C \sim C^{\prime}$, and $C$ is an H-complex, then $C^{\prime}$ is an H-complex.

From Lemmas 1.1 and 1.2, it follows that if we are considering an $H$-complex $C$ free over $R$, whose homology is of finite type, then, up to homotopy, we may consider $C$ to be of finite type. Thus for properties of homology, related to $H$-structure, we may consider $H$-complexes of finite type.

Lemma 1.3. If $C$ is an $H$-complex of finite type, then $C^{*}=\operatorname{Hom}(C, R)$ is an $H$-complex of finite type, and $C^{* *}=C$.

This follows easily from the usual properties of $\operatorname{Hom}(, R)$ on finitely generated free $R$-modules.

Note that if $d \equiv 0$ in $C, C$ an $H$-complex over $R$, then $C$ is a Hopf algebra over $R$ (since chain maps are homotopic only when they are equal, if $d \equiv 0$ ).

For any chain complex $C$, torsion free over the integers $Z$, and any prime $p$, we may define the Bockstein spectral sequence $\bmod p$ as follows:

If $C$ is torsion free over $Z$ then the sequence $0 \rightarrow C \stackrel{\leftrightarrow}{\rightarrow} C \stackrel{\dot{j}}{\rightarrow} C \otimes Z_{p} \rightarrow 0$ is exact, where $i(c)=p c, j=$ reduction $\bmod p$. The homology of this exact sequence of chain complexes forms an exact couple

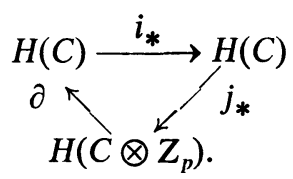

The spectral sequence of this exact couple $\left\{B^{r}\right\}, r \geqq 1$, is called the Bockstein spectral sequence of $C \bmod p$. A lengthy discussion is given in [7]. We use $\beta^{r}$ to denote the differential in $B^{r}$.

Lemma 1.4. Let $C$ be an $H$-complex free over $Z$, with $H(C)$ of finite type. Then the Bockstein spectral sequence of $C$ and $C^{*}$ are dual spectral sequences of Hopf algebras.

One may replace $C$ by an $H$-complex of finite type, and the rest of the proposition follows routinely.

We now introduce some notation and some properties of the Bockstein spectral sequence, which we will employ later. Let $C$ be a torsion free complex with $H(C)$ of finite type. 
The $r$ th derived couple is

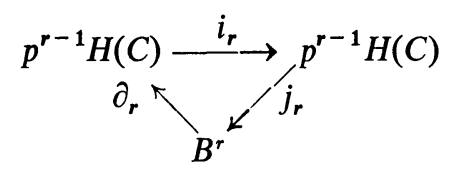

where $i_{r}(x)=p x$. We may define the map $k_{r}: H(C) \rightarrow B_{r}$ by $k_{r}(x)=j_{r}\left(p^{r-1} x\right)$, $\left(j_{1}=j_{*}\right)$.

LEMMA 1.5. Kernel $k_{r}=p H(C)+T_{r-1}$, where $T_{r-1}=$ subgroup of $H(C)$ of elements $x$ such that $p^{r-1} x=0$.

The proof of this lemma is routine, using properties of the Bockstein spectral sequence developed in [7].

2. Algebras of type $(n, 2 q)$. In this section we define some special Hopf algebras over $Z_{p}$ which we will use in the next section.

We define $A(n, 2 q)$ as follows: $A=A(n, 2 q)$ is generated as an algebra by $a_{0}, a_{1}, \cdots, a_{n}$, where $a_{i} \in A_{2 q p^{i}}$ and $A \cong \bigotimes_{i=0}^{n-1} Z_{p}\left[a_{i}\right] /\left(a_{i}^{p}\right) \otimes Z_{p}\left[a_{n}\right]$ as an algebra. The diagonal map $\psi: A \rightarrow A \otimes A$ is defined by

$$
\psi a_{i}=a_{i} \otimes 1+1 \otimes a_{i}+\Gamma_{i}
$$

where $\Gamma_{i}$ is defined inductively as follows:

Define $\Gamma_{0}=0$,

$$
\Gamma_{i+1}=\frac{1}{p}\left(\left(a_{i} \otimes 1+1 \otimes a_{i}\right)^{p}-a_{i}^{p} \otimes 1-1 \otimes a_{i}^{p}\right)+\left(a_{i} \otimes 1+1 \otimes a_{i}\right)^{p-1} \Gamma_{i} .
$$

It is easy to check that $A(n, 2 q)=A$ is an associative, commutative, co-associative and co-commutative Hopf algebra. It is easy to see also that $A_{m}=0$ if $2 q$ does not divide $m, A_{2 q k}=Z_{p}$. To find a generator of $A_{2 q k}$, expand the integer $k$ to the base $p$, i.e., $k=r_{0}+r_{1} p+\cdots+r_{s} p^{s}$. Then $\gamma_{k}(a)=a_{0}^{r_{0}} a_{1}^{r_{1}} \cdots a_{s}^{r_{s}}$ generates $A_{2 q k}$ where $a_{s}=a_{n}^{\nu^{s-n}}$ if $s \geqq n$.

There is a canonical map of Hopf algebras $r_{n}^{m}: A(m, 2 q) \rightarrow A(n, 2 q)$ for $m<n$ defined by sending $r_{n}^{m}\left(a_{i}\right)=a_{i}$ for $i \leqq m$.

If $B=Z_{p}[y]$ as a Hopf algebra $(\operatorname{dim} y=2 q)$, then $B^{*}=\operatorname{Hom}\left(B, Z_{p}\right)=\Gamma\left(y^{*}\right)$ as a Hopf algebra, where $\Gamma\left(y^{*}\right)$ is the twisted polynomial ring generated by $y^{*}$, i.e., $\Gamma$ is generated as a $Z_{p}$ module by $\gamma_{k}\left(y^{*}\right) \in \Gamma_{2 q k}$, multiplication is given by

$$
\left.\gamma_{k} \cdot \gamma_{l}=\begin{array}{c}
k+l \\
k
\end{array}\right) \gamma_{k+l}
$$

and the diagonal map $\Delta: \Gamma \rightarrow \Gamma \otimes \Gamma$ is given by $\Delta \gamma_{k}=\sum_{i+j=k} \gamma_{i} \otimes \gamma_{j}$.

LEMMA 2.1. $(A(n, 2 q))^{*}=Z_{p}\left[a_{0}^{*}\right] /\left(a_{0}^{* p^{n+1}}\right) \otimes \Gamma\left(\left(a_{n}^{p}\right)^{*}\right)$ as an algebra. 
Proof. As a coalgebra $A(n, 2 q) \cong \bigotimes_{i=0}^{n} Z_{p}\left[a_{i}\right] /\left(a_{i}^{p}\right) \otimes Z_{p}\left[a_{n}^{p}\right]$ with $a_{n}^{p}$ primitive, i.e., $\Delta a_{n}^{p}=a_{n}^{p} \otimes 1+1 \otimes a_{n}^{p}$. In the coalgebra $M=\otimes_{i=1}^{n} Z_{p}\left[a_{i}\right] /\left(a_{i}^{p}\right)$, the only primitive elements are multiples of $a_{0}$, so that $a_{0}^{*}$ generates $M^{*}$ since generators of $M^{*}$ are dual to primitive elements of $M$ (see [16]( $\left.{ }^{3}\right)$ ). Further, $M_{i}^{*}=0$ for $i \geqq 2 q p^{n+1}$ so that $M^{*}=Z_{p}\left[a_{0}^{*}\right] /\left(a_{0}^{* p^{n+1}}\right)$ and $(A(n, 2 q))^{*}=M^{*} \otimes \Gamma\left(\left(a_{n}^{p}\right)^{*}\right)$.

Lemma 2.2. Let $C$ be a Hopf algebra, $f: A(n, 2 q) \rightarrow C$ a map of Hopf algebras. If $f\left(a_{0}\right) \neq 0$, then $f$ is a monomorphism in dimensions $<2 q p^{n+1}$.

Proof. The lowest dimensional element in the kernel of a map of Hopf algebras is primitive. As noted in the proof of Lemma 2.1, the lowest dimensional primitive element of dimension $>2 q$ is in dimension $2 q p^{n+1}$, and the lemma follows.

LEMMA 2.3. Let $A^{(i)}$ be the Hopf subalgebra of $A(n, 2 q)$ generated by $a_{0}, \cdots, a_{i}$, $i<n$. Then $A_{m}^{(i)}=0$ for $m>2 q p^{i+1}-2 q$.

Proof. $A^{(i)}=\bigotimes_{k=0}^{i} Z_{p}\left[a_{k}\right] /\left(a_{k}^{p}\right)$ and $\left(Z_{p}\left[a_{k}\right] /\left(a_{k}^{p}\right)\right)_{m}=0$ if $m>2 q p^{k}(p-1)$. Hence $A_{m}^{(i)}=0$ if $m>\sum_{k=0}^{i} 2 q p^{k}(p-1)=2 q(p-1) \sum_{k=0}^{i} p^{k}=2 q\left(p^{i+1}-1\right)$.

LEMMA 2.4. $\Gamma_{i}^{2}=0$ in $A(n, 2 q) \otimes A(n, 2 q)$, for $i \leqq n$.

Proof. $\left(A^{(i-1)} \otimes A^{(i-1)}\right)_{m}=0$ for $m>4 q p^{i}-4 q$, by Lemma 2.3. Now $\Gamma_{i} \in\left(A^{(i-1)} \otimes A^{(i-1)}\right)_{2 q p^{i}}$ so that $\Gamma_{i}^{2} \in\left(A^{(i-1)} \otimes A^{(i-1)}\right)_{4 q p^{i}}$ and $4 q p^{i}>4 q p^{i}-4 q$ so that $\Gamma_{i}^{2}=0$.

3. Implications of higher torsion. In this section we shall prove" implication", theorems for higher terms $(r>1)$ of the Bockstein spectral sequence $B^{r}$ of an $H$-complex. These lead to various theorems about the homology of $H$-spaces in $\$ 4$.

Definition. Let $A$ be a Hopf algebra over $Z_{p}$. An element $x \in A_{m}$ is said to have $q$-implications if there exists a sequence $x=x_{0}, x_{1}, \cdots, x_{q}$, with $x_{i} \neq 0$ in $A_{m p}$, and for each $i<q$, either

(1) $x_{i+1}=x_{i}^{p}$ or

(2) there is an $\bar{x}_{i} \in A^{*}$ such that $\bar{x}_{i}\left(x_{i}\right) \neq 0$ and $\bar{x}_{i}^{p}\left(x_{i+1}\right) \neq 0$.

If an infinite such sequence exists, we say $x$ has $\infty$-implications.

If $A$ or $A^{*}$ is not associative, define $x^{n}$ by induction, $x^{n+1}=x^{n} \cdot x$.

An element $x$ of a Hopf algebra $A$ is primitive if $\psi x=x \otimes 1+1 \otimes x$, where $\psi: A \rightarrow A \otimes A$ is the diagonal map of $A$. We denote by $P(A)$ the space of primitive elements.

Let $C$ be an $H$-complex,

$$
i_{1}=1 \otimes \eta: C \rightarrow C \otimes C, \quad i_{2}=\eta \otimes 1: C \rightarrow C \otimes C,
$$

the injections of $C$ into the left and right factors of $C \otimes C$.

(3) One may also verify directly by computing that $\left(a_{i}^{*}\right)^{p}\left(a_{i+1}\right) \neq 0$, for $0 \leqq i<n$, so that $a_{0}^{*}$ generates $(A(n, 2 q)) *$ in dimensions $<2 q p^{n+1}$. 
Then for any element $u \in H(C \otimes G), \psi_{*} u=i_{1 *} u+i_{2 *} u+Q$, where $Q \in$ kernel of $\pi_{1 *}+\pi_{2 *}: H(C \otimes C) \rightarrow H(C)+H(C)$, where $\pi_{1}=1 \otimes \varepsilon, \quad \pi_{2}=\varepsilon \otimes 1$ are projections on the left and right factors of $C \otimes C$. This follows from the fact that $\pi_{j} \circ i_{k}=\delta_{j k} \cdot 1,\left(\delta_{j k}=0\right.$ if $j \neq k,=1$ if $\left.j=k\right)$.

Let $\gamma: H\left(C \otimes Z_{p}\right) \rightarrow H\left(C \otimes Z_{p^{2}}\right)$ be induced from the injection $Z_{p} \rightarrow Z_{p^{2}}$.

Lemma 3.1. Let $I=$ image $\gamma \subseteq H\left(C \otimes Z_{p^{2}}\right)$, where $C$ is a differential algebra. Then $I$ is an ideal, and $I^{2}=0$.

Proof. An element $x \in H\left(C \otimes Z_{p^{2}}\right)$ is in $I$ if and only if there is a chain $c \in C$, with $p c$ representing $x$. Then the lemma follows by a direct chain argument.

Lemma 3.2. Let $C$ and $C^{\prime}$ be differential algebras, $i: C \rightarrow C \otimes C^{\prime}$, $i^{\prime}: C^{\prime} \rightarrow C \otimes C^{\prime}$ the two injections $(i(c)=c \otimes 1$, etc. $)$. If $u \in H(C \otimes A)$, $u^{\prime} \in H\left(C^{\prime} \otimes A\right)$, A some commutative ring, then

$$
\left(i(u)+i^{\prime}\left(u^{\prime}\right)\right)^{p}=\sum_{j+k=p}\left(\begin{array}{l}
p \\
j
\end{array}\right)(i(u))^{j}\left(i^{\prime}\left(u^{\prime}\right)\right)^{k}
$$

in $H\left(C \otimes C^{\prime} \otimes A\right)$.

Proof. By definition of the algebra structure of $C \otimes C^{\prime}$, the elements $(i(u))^{j}$ and $\left(i^{\prime}\left(u^{\prime}\right)\right)^{k}$ commute. Then the lemma follows by induction on $p$ as in the associative case.

We denote by $q: H\left(C \otimes Z_{p^{2}}\right) \rightarrow H\left(C \otimes Z_{p}\right)$ the map induced by reduction $\bmod p$, so that we have the exact sequence

$$
\ldots \stackrel{\beta_{1}}{\rightarrow} H_{k}\left(C \otimes Z_{p}\right) \stackrel{\gamma}{\rightarrow} H_{k}\left(C \otimes Z_{p^{2}}\right) \stackrel{q}{\rightarrow} H_{k}\left(C \otimes Z_{p}\right) \stackrel{\beta_{1}}{\rightarrow} \cdots .
$$

THEOREM 3.3. Let $C$ be an H-complex, with $H(C)$ of finite type, $H_{0}(C)=Z$, and suppose $u \in H_{2 m}\left(C \otimes Z_{p^{2}}\right)$ with $p u \neq 0$ and $q(u) \in P\left(H\left(C \otimes Z_{p}\right)\right)$. Then $q(u)$ has 1-implication in $H\left(C \otimes Z_{p}\right)$.

(Note that there are no requirements of associativity or commutativity.)

$\operatorname{Proof}\left({ }^{4}\right) . \quad \psi_{*} u=u_{1}+u_{2}+Q$, where $u_{1}=i_{1 *} u, u_{2}=i_{2 *} u, Q \in($ kernel $q)$ $\cap\left(\operatorname{kernel}\left(\pi_{1 *}+\pi_{2 *}\right)\right)$, since $q(u)$ is primitive. Then $Q \in I$, so $Q=\gamma\left(Q^{\prime}\right)$, $Q^{\prime} \in H\left(C \otimes C \otimes Z_{p}\right)$.

Now

$$
\begin{aligned}
\psi_{*}\left(u^{p}\right) & =\left(\psi_{*}(u)\right)^{p}=\left(u_{1}+u_{2}+Q\right)^{p} \\
& =\left(u_{1}+u_{2}\right)^{p}+\sum_{l}\left(u_{1}+u_{2}\right)^{p-l} Q\left(u_{1}+u_{2}\right)^{l-1}+M,
\end{aligned}
$$

(4) Caution: In many of the calculations below we have ignored the association of products (as in (3.4), (3.6)). Some of these terms should have parentheses distributed on the factors, but since the arguments are on the dimension of these products, the ambiguity does not affect the result. If one tries to strengthen this theorem (analogously to Theorem 3.11), the difficulties of nonassociativity and noncommutativity magnify. 
where $M$ involves two or more factors $Q$. It follows from Lemma 3.1 that $M=0$ (this does not require associativity). Hence, by Lemma 3.2,

$$
\begin{aligned}
\psi_{*}\left(u^{p}\right)= & u_{1}^{p}+u_{2}^{p}+\sum_{i+j=p ; i, j>0}\left(\begin{array}{l}
p \\
i
\end{array}\right) u_{1}^{i} u_{2}^{j} \\
& +\sum_{l}\left(u_{1}+u_{2}\right)^{p-l} Q\left(u_{1}+u_{2}\right)^{l-1} .
\end{aligned}
$$

By a direct chain argument it is easy to show:

Lemma 3.5. Let $C$ be a differential algebra, $x \in H\left(C \otimes Z_{p^{2}}\right), z \in H\left(C \otimes Z_{p}\right)$. Then $x \cdot \gamma(z)=\gamma(\bar{x} \cdot z), \gamma(z) \cdot x=\gamma(z \cdot \bar{x})$, where $\bar{x}=q(x)$.

Now $q\left(u_{1}\right)=\bar{u} \otimes 1, q\left(u_{2}\right)=1 \otimes \bar{u}$, where $\bar{u}=q(u)$, and let $Q^{\prime}=\sum_{i} s_{i} \otimes t_{i}$ $\left(Q=\gamma\left(Q^{\prime}\right)\right), s_{i}, t_{i} \in H\left(C \otimes Z_{p}\right)$. Since $Q \in \operatorname{kernel}\left(\pi_{1 *}+\pi_{2 *}\right)$, it follows that we may choose $Q^{\prime}$ so that $\operatorname{dim} s_{i}>0, \operatorname{dim} t_{i}>0$, for all $i\left(\operatorname{dim} s_{i}+\operatorname{dim} t_{i}=\operatorname{dim} u=2 m\right)$.

If $\bar{u}^{p}=q\left(u^{p}\right) \neq 0$, then $\bar{u}, \bar{u}^{p}$ is a 1 -implication sequence. So assume $\bar{u}^{p}=0$, so that $u^{p}=\gamma(v), v \in H\left(C \otimes Z_{p}\right)$.

Note that $p u=\gamma(\bar{u})$. Define $a_{i}=(1 / p)\left(\begin{array}{l}p \\ i\end{array}\right)$, and note that $a_{i} \neq 0 \bmod p$. Then from (3.4) and (3.5), we get:

$$
\begin{aligned}
\psi_{*}(\gamma(v))= & \gamma\left(v_{1}\right)+\gamma\left(v_{2}\right)+\gamma\left(\sum a_{i} \bar{u}^{p-i} \otimes \bar{u}^{i}\right) \\
& +\gamma\left(\sum_{l}\left(\bar{u}_{1}+\bar{u}_{2}\right)^{p-l} Q^{\prime}\left(\bar{u}_{1}+\bar{u}_{2}\right)^{l-1}\right) .
\end{aligned}
$$

Hence,

$$
\begin{aligned}
\psi_{*}(v)= & v \otimes 1+1 \otimes v+\sum a_{i} \bar{u}^{p-i} \otimes \bar{u}^{i} \\
& +\sum_{l}\left(\bar{u}_{1}+\bar{u}_{2}\right)^{p-l} Q^{\prime}\left(\bar{u}_{1}+\bar{u}_{2}\right)^{l-1}+K,
\end{aligned}
$$

where $K \in$ kernel $\gamma=$ image $\beta_{1}$. Now the terms involving $Q^{\prime}$ are sums of terms of the form $\left(\bar{u}^{a} s_{i} \bar{u}^{b}\right) \otimes\left(\bar{u}^{c} t_{i} \bar{u}^{d}\right)$ where $a+b+c+d=p-1$. Since $0<\operatorname{dim} s_{i}<2 m, 0<\operatorname{dim} t_{i}<2 m$, and $\operatorname{dim} \bar{u}=2 m$, no term of this form is in $H_{2 m(p-1)}\left(C \otimes Z_{p}\right) \otimes H_{2 m}\left(C \otimes Z_{p}\right)$. Let $x \in H^{2 m}\left(C \otimes Z_{p}\right)$ be such that $x(\bar{u}) \neq 0$ and $\beta_{1} x=0$, which is possible since $p u \neq 0$. Then, from (3.6)

$$
\begin{aligned}
x^{p}(v) & =\left(x^{p-1} \otimes x\right)\left(\psi_{*}(v)\right) \\
& =a_{1} x^{p-1}\left(\bar{u}^{p-1}\right) x(\bar{u})+\left(x^{p-1} \otimes x\right)(K),
\end{aligned}
$$

since no other elements can have the right dimension. But

$$
\left(x^{p-1} \otimes x\right)(K)=\left(x^{p-1} \otimes x\right)\left(\beta_{1} K^{\prime}\right)=\left(\beta_{1}\left(x^{p-1} \otimes x\right)\right)\left(K^{\prime}\right)=0 .
$$

But it is easy to show by induction, using the primitivity of $\bar{u}$, that $x^{p-1}\left(\bar{u}^{p-1}\right)=(p-1) !(x(\bar{u}))^{p-1} \neq 0$. Hence $x^{p}(v) \neq 0$ and $x^{p} \neq 0$, which completes the proof. 
Now we will make stronger assumptions on the $H$-complex $C$ and, using machinery of $\$ 2$, prove a result (Theorem 3.7) which will have many useful applications. For an abelian group $M$, denote by $M_{p}$ the $p$-primary subgroup of $M$.

THEOREM 3.7. Let $C$ be an $H$-complex with $H(C)$ of finite type, $H_{0}(C)=Z$, and suppose that $H(C)$ is an associative, commutative algebra. Let $c_{0}, \cdots, c_{n} \in H(C)_{p}$, such that $p c_{i+1}=c_{i}^{p}, 0 \leqq i<n$, with $k_{r}\left(c_{n}\right)=j_{r}\left(p^{r-1} c_{n}\right) \neq 0$. Supposef $\left(a_{i}\right)=k_{s}\left(c_{i}\right)$, for $0 \leqq i \leqq n$, defines a homomorphism of Hopf algebras, $f: A(n, 2 q) \rightarrow B^{s}, s<r$. Then

(1) $p^{r-2} c_{n}^{p} \neq 0$;

(2) if $k_{s}\left(c_{n}^{p}\right)=0$, then $c_{n}^{p}=p c_{n+1}$ and $f^{\prime}\left(a_{i}\right)=k_{s+1}\left(c_{i}\right), 0 \leqq i \leqq n+1$, defines a map of Hopf algebras $f^{\prime}: A(n+1,2 q) \rightarrow B^{s+1}$.

(See $\$ 1$ for definitions of $j_{r}$ and $k_{s}$.)

Proof. Let us define $G_{j} \in H(C \otimes C)$ in a similar way to $\Gamma_{j} \in A(n, 2 q) \otimes A(n, 2 q)$, i.e., let $G_{0}=0$,

$$
G_{j+1}=\frac{1}{p} \sum_{k=1}^{p-1}\left(\begin{array}{l}
p \\
k
\end{array}\right) c_{j}^{p-k} \otimes c_{j}^{k}+\left(c_{j} \otimes 1+1 \otimes c_{j}\right)^{p-1} G_{j},
$$

where we are considering $H(C) \otimes H(C)$ as a subalgebra of $H(C \otimes C)$ (which we may do by the Künneth formula). Then since $f: A(n, 2 q) \rightarrow B^{s}$ is a Hopf algebra map and $f\left(\Gamma_{j}\right)=k_{s}\left(G_{j}\right)$, it follows that $\psi\left(c_{n}\right)=c_{n} \otimes 1+1 \otimes c_{n}+G_{n}+A+p E$, where $p^{s-1} A=0, A, E \in H(C \otimes C$ ) (see Lemma 1.5).

Then

$$
\begin{aligned}
\psi\left(c_{n}^{p}\right)= & \left(\psi c_{n}\right)^{p}=\left(c_{n} \otimes 1+1 \otimes c_{n}+G_{n}+A+p E\right)^{p} \\
= & \left(c_{n} \otimes 1+1 \otimes c_{n}+G_{n}+A\right)^{p} \\
& +\sum_{k=1}^{p}\left(\begin{array}{l}
p \\
k
\end{array}\right)\left(c_{n} \otimes 1+1 \otimes c_{n}+G_{n}+A\right)^{p-k}(p E)^{k} \\
= & \left(c_{n} \otimes 1+1 \otimes c_{n}+G_{n}+A\right)^{p}+p^{2} Q \\
= & c_{n}^{p} \otimes 1+1 \otimes c_{n}^{p}+p G_{n+1}+\sum_{k=2}^{p-1}\left(\begin{array}{l}
p \\
k
\end{array}\right)\left(c_{n} \otimes 1+1 \otimes c_{n}\right)^{p-k} G_{n}^{k} \\
& +G_{n}^{p}+\sum_{l=1}^{p}\left(\begin{array}{l}
p \\
l
\end{array}\right)\left(c_{n} \otimes 1+1 \otimes c_{n}+G_{n}\right)^{p-l} A^{l}+p^{2} Q,
\end{aligned}
$$

where at the third step we have collected terms with a factor of $p^{2}$ into $p^{2} Q$.

LEMMA 3.8. $G_{n}^{2} \in p H(C \otimes C)$.

Proof. If we set $g\left(a_{i}\right)=j_{*}\left(c_{i}\right) \in H\left(C \otimes Z_{p}\right)$, for $0 \leqq i \leqq n$, this defines a map of algebras, $g: A(n, 2 q) \rightarrow H\left(C \otimes Z_{p}\right)$, and $g\left(\Gamma_{n}\right)=j_{*}\left(G_{n}\right)$. But $\Gamma_{n}^{2}=0$, by Lemma 2.4 so that $j_{*}\left(G_{n}^{2}\right)=0$, and thus $G_{n}^{2} \in p H(C \otimes C)$. 
LEMMA 3.9. $G_{n}^{p} \in p^{2} H(C \otimes C)$.

Proof. Let $D$ be the subalgebra of $H(C)$ generated by $c_{0}, \cdots, c_{n-1}$, and let $\zeta(x)=x^{p}$. Then, since $j_{*}(D)=f\left(A^{\prime}\right)$, where $A^{\prime}=$ subalgebra of $A(n, 2 q)$ generated by $a_{0}, \cdots, a_{n-1}$, and $\zeta\left(A^{\prime}\right)=0$, it follows that $\zeta(D) \subseteq p H(C)$. Also $A_{m}^{\prime}=0$ for $m>2 q p^{n}-2 q$ by Lemma 2.3 , so that $D_{m} \subseteq p H(C)$ for $m>2 q p^{n}-2 q$, and hence $(D \otimes D)_{l} \subseteq p H(C \otimes C)$ if $l>4 q p^{n}-4 q$. Now $G_{n} \in(D \otimes D)_{2 q p^{n}}$. We shall show that for any element $x \in(D \otimes D)_{2 q p^{n}}, x^{p} \in p^{2} H(C \otimes C)$. If $x$ is an element of the form $a \otimes b \in D_{i} \otimes D_{j}, i+j=2 q p^{n}$, then $x^{p}=a^{p} \otimes b^{p}$ and $a^{p}, b^{p} \in p H(C)$, so $x^{p} \in p^{2} H(C \otimes C)$. If $x, y \in(D \otimes D)_{2 q p^{n}}$ and $x^{p}, y^{p} \in p^{2} H(C \otimes C)$, then

$$
(x+y)^{p}=x^{p}+y^{p}+\sum_{k=1}^{p-1}\left(\begin{array}{l}
p \\
k
\end{array}\right) x^{p-k} y^{k}
$$

and $x^{p-k} y^{k} \in(D \otimes D)_{2 q p^{n+1}} \subseteq p H(C \otimes C)$. Hence, each term in $(x+y)^{p}$ is a multiple of $p^{2}$. Since elements $a \otimes b \in D_{i} \otimes D_{j}, i+j=2 q p^{n}$, form a basis for $(D \otimes D)_{2 q p^{n}}$, the lemma now follows.

It follows from Lemmas 3.8 and 3.9 that

$$
\psi\left(c_{n}^{p}\right)=c_{n}^{p} \otimes 1+1 \otimes c_{n}^{p}+p G_{n+1}+\bar{A}+p^{2} Q^{\prime},
$$

where $p^{s-1} \bar{A}=0(\bar{A}=$ sum of all terms with a factor $A)$. Multiplying by $p^{r-2}$, we get

$$
p^{r-2} \psi\left(c_{n}^{p}\right)=\psi\left(p^{r-2} c_{n}^{p}\right)=p^{r-2} c_{n}^{p} \otimes 1+1 \otimes\left(p^{r-2} c_{n}^{p}\right)+p^{r-1} G_{n+1}+p^{r} Q^{\prime},
$$

$\left(p^{r-2} \bar{A}=0\right.$ since $\left.r-2 \geqq s-1\right)$. Since $j_{r}\left(p^{r-1} c_{n}\right) \neq 0$, it follows that $j_{r}\left(p^{r-1} G_{n}\right) \neq 0$, since $j_{r}\left(p^{r-1} c_{n}\right)=k_{r}\left(c_{n}\right), j_{r}\left(p^{r-1} G_{n}\right)=k_{r}\left(G_{n}\right)$, and $k_{r}\left(G_{n}\right)=k_{r}\left(c_{n}\right) \otimes k_{r}\left(c_{n}^{p-1}\right)+$ terms of different degree in the first factor, hence is nonzero. But $j_{r}\left(p^{r} Q^{\prime}\right)=0$, so that $j_{r}\left(p^{r-2} \psi\left(c_{n}^{p}\right)\right) \neq 0$ and hence $p^{r-2} c_{n}^{p} \neq 0$, which proves (1).

If $j_{*} c_{n}^{p}=k_{1} c^{p} \neq 0$, then since order $c^{p} \geqq p^{r-1}$ by (1) and $s<r$, it follows that $k_{s}\left(c_{n}^{p}\right) \neq 0$. Hence if $k_{s}\left(c_{n}^{p}\right)=0$, then $j_{*}\left(c_{n}^{p}\right)=0$, so that $c_{n}^{p}=p c_{n+1}$, for some element $c_{n+1} \in H_{2 q p^{n+1}}(C)$.

We use (3.10) to compute the diagonal map on $c_{n+1}$, since $p c_{n+1}=c_{n}^{p}$, and we get

$$
\psi\left(c_{n+1}\right)=c_{n+1} \otimes 1+1 \otimes c_{n+1}+G_{n+1}+A_{1}+p Q^{\prime},
$$

where $A_{1} \in H(C \otimes C)$ is such that $p A_{1}=\bar{A}$, so that since $p^{s-1} \bar{A}=0$, we have that $p^{s} A_{1}=0$. Hence $k_{s+1} A_{1}=0$. If we set $f^{\prime}\left(a_{i}\right)=k_{s+1}\left(c_{i}\right)$, for $0 \leqq i \leqq n+1$, this defines a map of algebras, $f^{\prime}: A(n+1,2 q) \rightarrow B^{s+1}$, and $f^{\prime} \circ \psi=\psi \circ f^{\prime}$, since $k_{s}\left(G_{n+1}\right)=f^{\prime}\left(\Gamma_{n+1}\right), k_{s+1}\left(p Q^{\prime}\right)=k_{s+1}\left(A_{1}\right)=0$. Hence $f^{\prime}$ defines a map of Hopf algebras, which proves (2), completing the proof of Theorem 3.7.

REMARK. If $k_{s}\left(c_{n}^{p}\right)=0$, it follows that $p^{r-1} c_{n+1} \neq 0$, since $p^{r-2} c_{n}^{p} \neq 0$ by (1).

From Theorem 3.7, we now derive a result which is in a more convenient form for applications. 
THEOREM 3.11. Let $C$ be an $H$-complex, with $H(C)$ of finite type and $H_{0}(C)=Z$, and suppose that $H(C)$ is commutative and associative. Let $x \in H_{2 q}(C)_{p}$, such that $k_{r}(x) \neq 0$, and $k_{s}(x) \in P\left(B^{s}\right), s<r$. Then $k_{s}(x)$ has $(r-s)$-implications in $B^{s}$.

Proof. If we set $x=c_{0}$, the hypothesis of Theorem 3.7 is satisfied for $n=0$. Proceeding by induction on $(r-s)$, if $k_{s} c_{0}^{p} \neq 0$, then $k_{s} c_{0}^{p} \in P\left(B^{s}\right)$ and $k_{r-1}\left(c_{0}^{p}\right) \neq 0$, since $p^{r-2} c_{0}^{p} \neq 0$ by (1) in Theorem 3.7. Hence, by induction, $k_{s}\left(c_{0}^{p}\right)$ has $(r-s-1)$ implications, so that $k_{s}\left(c_{0}\right)$ has $(r-s)$-implications. If $k_{s}\left(c_{0}^{p}\right)=0$, then by (2) in Theorem 3.7, $c_{0}^{p}=p c_{1}$, and $f\left(a_{i}\right)=k_{s+1}\left(c_{i}\right), i=0,1$, defines a map $f: A(1,2 q) \rightarrow B^{s+1}$. Assume (by another induction) that we have defined a map $f: A(j, 2 q) \rightarrow B^{s+j}, \quad j<r-s, \quad$ by $f\left(a_{i}\right)=k_{s+j}\left(c_{i}\right), \quad c_{i}^{p}=p c_{i+1}, \quad k_{r}\left(c_{j}\right) \neq 0 . \quad$ If $f\left(a_{j}^{p}\right)=\left(k_{s+j}\left(c_{j}\right)\right)^{p} \neq 0$, then it is primitive, since $a_{j}^{p} \in P(A(j, 2 q))$, and $k_{r-1}\left(c_{j}^{p}\right) \neq 0$ by $(3.7(1))$, so that our first induction hypothesis applies and $k_{s+j}\left(c_{j}^{p}\right)$ has $(r-s-j-1)$-implications.

Now we show that $k_{s+j}\left(c_{0}\right), \cdots, k_{s+j}\left(c_{j}\right)$ is a $j$-implication sequence for $k_{s+j}\left(c_{0}\right)$. Since $\left(k_{s+j}\left(c_{i}\right)\right)^{p}=0$, for $0 \leqq i \leqq j$, we must show that for each $i<j$, there is a $y_{i} \in B_{s+j}, y_{i}\left(k_{s+j}\left(c_{i}\right)\right) \neq 0$, and $y_{i}^{p}\left(k_{s+j}\left(c_{i+1}\right)\right) \neq 0$. But $y_{i}\left(k_{s+j}\left(c_{i}\right)\right) \neq 0$ implies that $f^{*}\left(y_{i}\right)=a_{i}^{*}=$ a generator of $(A(j, 2 q))^{2 q p^{i}}$. By Lemma 2.2, $\left(a_{i}^{*}\right)^{p}\left(a_{i+1}\right) \neq 0$ (since $\left.a_{i}^{p} \neq 0\right)$, and hence $\left(f^{*}\left(y_{i}\right)\right)^{p}\left(a_{i+1}\right)=y_{i}^{p}\left(f_{*}\left(a_{i+1}\right)\right)=y_{i}^{p}\left(k_{s+j}\left(c_{i+1}\right)\right) \neq 0$. It follows that $k_{s+j}\left(c_{0}\right), \cdots, k_{s+j}\left(c_{j}\right)$ is a $j$-implication sequence for $k_{s+j}\left(c_{0}\right)$. Then since $k_{s+j}\left(c_{j}^{p}\right)$ has $(r-s-j-1)$-implications in $B^{s+j}, k_{s+j}\left(c_{j}\right)$ has $(r-s-j)$-implications in $B^{s+j}$; hence $k_{s}\left(c_{j}\right)$ has $(r-s-j)$-implications in $B^{s}$, so that finally $k_{s}\left(c_{0}\right)$ has $(r-s)$-implications in $B^{s}$ (cf. [7, Lemma 6.4]).

If $k_{s+j}\left(c_{j}^{\mu}\right)=0$, then Theorem 3.7(2) applies and we get the second induction hypothesis for $j+1$, and the theorem follows.

REMARK. The implication sequence $x_{0}=k_{s}(x), x_{1}, \cdots, x_{r-s}$, constructed by the above proof has the slightly stronger property that if $x_{i}^{p}=0, i<r-s$, then for any $\bar{x}_{i} \in B_{s}$, with $\bar{x}_{i}\left(x_{i}\right) \neq 0$, we have $\bar{x}_{i}^{p}\left(x_{i+1}\right) \neq 0$.

THEOREM 3.12. Let $C$ be an $H$-complex with $H(C)$ of finite type, $H_{0}(C)=Z$ and $H(C)$ associative and commutative. Suppose $\beta^{m} \equiv 0$ for some $m$, so that $B^{m+1}=B^{m}$, in the Bockstein spectral sequence of $C \bmod p$. If $x \in H_{2 q}(C)_{p}$, with $k_{r}(x) \neq 0, k_{s}(x) \in P\left(B^{s}\right), s \leqq m<r$, then $k_{s}(x)$ has $\infty$-implications.

Proof. We use the same proof as Theorem 3.11, and note that at each stage of the proof we have a map $f: A(n, 2 l) \rightarrow B^{s+j}$, with $f\left(a_{n}\right)=k_{s+j}\left(c_{n}\right), k_{r-i}\left(c_{n}\right) \neq 0$, $s+j<r-i$, and we apply Theorem 3.7. At the next stage in the proof we either have a map $f^{\prime}: A(n+1,2 l) \rightarrow B^{s+j+1}$ with $k_{r-i}\left(c_{n+1}\right) \neq 0, f\left(a_{n+1}\right)=k_{s+j+1}\left(c_{n+1}\right)$, or an element $y=k_{s+j}\left(c_{n}^{p}\right) \in P\left(B^{s+j}\right)$ and $k_{r-i-1}\left(c_{n}\right) \neq 0$. The process stops in the proof of Theorem 3.11 because finally either $r-i=s+j+1$ or $r-i-1=s+j$. In other words, the indices come together, i.e., we get to the situation where we have a map $f^{\prime \prime}: A(s, 2 t) \rightarrow B^{u}$ with $f^{\prime \prime}\left(a_{s}\right)=k_{u}\left(c_{s}\right)$ and $k_{v}\left(c_{s}\right) \neq 0$, but $v=u$. But if 
$\beta^{m} \equiv 0$ so that $B^{m}=B^{m+1}$ and $s \leqq m<r$, then maps into $B^{m}$ are the same as maps into $B^{m+1}$. Hence $s+j$ can always be kept $\leqq m$, and $r-i$ can be kept $\geqq m+1$, so the indices are kept apart. Hence the argument does not stop and we get an $\infty$-implication sequence.

Corollary 3.13. Let $C$ be an associative commutative $H$-complex with $d \equiv 0$ (i.e., $C$ is a free Hopf algebra over $Z)$. Then an element $x \in P\left(C_{2 q} \otimes Z_{p}\right)$ has $\infty$-implications.

Proof. Apply Theorem 3.12, using the fact that $\beta^{1} \equiv 0\left(C \otimes Z_{p}=H\left(C \otimes Z_{p}\right)\right.$ $=B^{1}=B^{2}=\cdots=B^{\infty}$ in this case).

4. Applications to $H$-spaces. In this section we apply the results of $\S 3$ to $H$-spaces, obtaining "implication" theorems we shall apply in $\$ 5$ and obtain also some theorems on finite dimensional $H$-spaces.

Since the chains or cochains of an $H$-space are $H$-complexes, we get:

THEOREM 4.1. Let $X$ be a connected $H$-space with $H_{*}(X)$ of finite type. If $x \in H_{2 m}\left(X ; Z_{p^{2}}\right), p x \neq 0$ and $q(x) \in P\left(H_{2 m}\left(X ; Z_{p}\right)\right)(q=$ reduction $\bmod p)$, then $q(x)$ has 1-implication in $H_{*}\left(X ; Z_{p}\right)$.

This is immediate from Theorem 3.3.

Corollary 4.2. Let $X$ be a connected $H$-space with $H_{*}(X)$ of finite type. Let $x \in P\left(H_{2 m}\left(X ; Z_{p}\right)\right)$, and suppose $\beta_{1} x=0$. Then $x$ has 1-implication in $H_{*}\left(X ; Z_{p}\right)$.

REMARK. This shows that in [9, Theorem 6.7] $l \geqq 2$.

Proof. If $x \in$ image $\beta_{1}$, then $x$ has $\infty$-implications by [7, Theorem 6.1] (8.1). If $x \notin$ image $\beta_{1}$, then by [7, Proposition 1.2] $\{x\}=\{q(u)\}$ in $B^{2}\left(B^{r}=\right.$ the homology Bockstein spectral sequence of $X \bmod p)$. Then $x-q(u)=\beta_{1} z$. But $\beta_{1}=q \partial_{1}$, so that $x=q(u)+q\left(\partial_{1} z\right)$. Also $p\left(u+\partial_{1} z\right)=p u \neq 0$, since $\{q(u)\} \neq 0$ in $B^{2}$. Hence $u+\partial_{1} z$ satisfies the hypothesis of Theorem 4.1 , so $q\left(u+\partial_{1} z\right)=x$ has 1-implication in $H_{*}\left(X ; Z_{p}\right)$. Q.E.D.

THEOREM 4.3. Let $X$ be a connected $H$-space with $H_{*}(X)$ of finite type, $\left\{B_{r}\right\}$ the cohomology Bockstein spectral sequence $\bmod p$ of $X$. Let $x \in H^{2 q}(X)_{p}$ such that $k_{r}(x) \neq 0$, and $k_{s}(x) \in P\left(B_{s}\right), s<r$. Then $k_{s}(x)$ has $(r-s)$-implications in $B_{s}$.

This follows from Theorem 3.11 since $H^{*}(X)$ is associative and commutative.

Corollary 4.4. Let $X$ be a connected $H$-space, $H_{*}(X)$ of finite type. If $x \in P\left(B_{s}^{2 q}\right)$, $\beta_{j} x=0$ for $s \leqq j<r$, and $\{x\} \neq 0$ in $B_{r}$, then $x$ has $(r-s)$-implications in $B_{s}$.

This follows from Theorem 4.3 and [7, Theorem 6.12] (8.2), by an argument similar to that of Corollary 4.2 . 
For $s=1, r=2,(4.4)$ was proved earlier by J. C. Moore (in unpublished work) by a different method.

THEOREM 4.5. Let $X$ be a connected $H$-space with $H_{*}(X)$ of finite type, and suppose $\beta_{m} \equiv 0$ for some $m$, so that $B_{m}=B_{m+1}$ (the cohomology Bockstein spectral sequence $\bmod p)$. If $x \in P\left(B_{s}^{2 q}\right), \beta_{j} x=0$ for $s \leqq j<r, s \leqq m<r,\{x\} \neq 0$ in $B_{r}$, then $x$ has $\infty$-implications in $B_{s}$.

This follows from Theorem 3.12 and [7, Theorem 6.12], (8.2).

Corollary 4.6. Let $X$ be a connected $H$-space, $H_{*}(X)$ of finite type, and suppose $H_{i}\left(X ; Z_{p}\right)=0$ for $i$ large, i.e., $H_{*}\left(X ; Z_{p}\right)$ is finitely generated. If $\beta_{m} \equiv 0$, then $B_{m}=B_{\infty}$, i.e., $\beta_{k} \equiv 0$ for $k \geqq m$.

Proof. Let $j$ be the smallest integer $>m$, such that $\beta_{j} \not \equiv 0$, and let $x=\beta_{j} y$ be a lowest dimensional element in image $\beta_{j}$. Then $x$ is primitive since it is a lowest dimensional element in image $\beta_{j}$. The dimension of $x$ is even, for if it were odd, then a lowest dimensional element $z$ in image $\beta_{j}$ (in the homology Bockstein spectral sequence) would be a primitive element in an even dimension. Then $z$ would have $\infty$-implications by [7, Theorem 6.1] (8.1) which is impossible, since $H_{*}\left(X ; Z_{p}\right)$ is assumed finitely generated. Then, since $B_{m}=B_{m+1}=\cdots=B_{j}$, there is an element $x^{\prime} \in P\left(B_{m}^{2 q}\right)$ with $\left\{x^{\prime}\right\}=x \in B_{j}, j>m$. By Theorem 4.5, $x^{\prime}$ has $\infty$-implications, which contradicts the finite generation of $H_{*}\left(X ; Z_{p}\right)$. Hence $\beta_{j} \equiv 0$ for all $j \geqq m$, which proves the corollary.

From Corollary 3.13 , we get the following two theorems:

THEOREM 4.7. Let $X$ be a connected $H$-space with $H_{*}(X)$ of finite type. If $x \in P\left(B_{\infty}^{2 m}\right)$, then $x$ has $\infty$-implications in $B_{\infty}$ (cohomology Bockstein spectral sequence).

ThEOREM 4.8. Let $X$ be a connected $H$-space, with $H_{*}(X)$ of finite type, and suppose $H_{*}(X) /$ torsion is an associative, commutative algebra. If $x \in P\left(B_{2 m}^{\infty}\right)$, then $x$ has $\infty$-implications in $B^{\infty}$.

These theorems follow immediately from Corollary 3.13, since $B_{\infty}=\left(H^{*}(X) /\right.$ torsion $) \otimes Z_{p}, B^{\infty}=\left(H_{*}(X) /\right.$ torsion $) \otimes Z_{p}$, and $H^{*}(X) /$ torsion, $H_{*}(X) /$ torsion, are free Hopf algebras over $Z$, which are associative and commutative under the above hypotheses.

THEOREM 4.9. Let $X$ be a connected $H$-space, with $H_{*}(X)$ of finite type and $H_{*}\left(X ; Z_{p}\right)$ finitely generated. If $H^{*}\left(X ; Z_{p}\right)$ is primitively generated, then $\beta_{2} \equiv 0$ in $B_{2}, B_{2}=B_{\infty}$ and all elements of p-torsion in $H_{*}(X)$ have order exactly $p$.

Proof. According to $[16$, Theorem 4.9], (see also $[9, \S 7]), H^{*}\left(X ; Z_{p}\right)$ being primitively generated implies that $H_{*}\left(X ; Z_{p}\right)$ is associative, commutative, and 
$u^{p}=0$ for any positive dimensional $u \in H_{*}\left(X ; Z_{p}\right)$. Hence if an element $x \in H^{*}\left(X ; Z_{p}\right)$ has 1-implication, then $x^{p} \neq 0$.

Let $x=\beta_{2} y$ be a lowest dimensional element in image $\beta_{2}$, so $\operatorname{dim} x=2 q$ by [9, Lemma 6.5]. Let $x^{\prime} \in H^{*}\left(X ; Z_{p}\right)$ such that $\left\{x^{\prime}\right\}=x \in B_{2}$. It follows that $x^{\prime}$ may be chosen to beindecomposable (see [9, Lemma 6.5]). Then $x^{\prime}$ may be chosen primitive in $H^{*}\left(X ; Z_{p}\right)$, since $H^{*}\left(X ; Z_{p}\right)$ is primitively generated. From Corollary 4.4 , it follows that $x^{\prime}$ has 1 -implication so that $\left(x^{\prime}\right)^{p} \neq 0$. If $\left\{\left(x^{\prime}\right)^{p}\right\} \neq 0$ in $B_{2}$, then, since $\left(x^{\prime}\right)^{p} \in P\left(H^{*}\left(X ; Z_{p}\right)\right),\left(x^{\prime}\right)^{p}$ has 1-implication in $H^{*}\left(X ; Z_{p}\right)$, and $\left(x^{\prime}\right)^{p 2} \neq 0$. Let $h=p^{k}$ be the smallest power of $p$ such that if $\left(x^{\prime}\right)^{h}=a,\{a\} \neq 0$ in $B_{2},\left\{a^{p}\right\}=0$ in $B_{2}, a^{p} \neq 0$ in $B_{1}=H^{*}\left(X ; Z_{p}\right)$.

Hence $a^{p}=\beta_{1} b, b \in H^{*}\left(X ; Z_{p}\right)$.

LEMMA 4.10. If $\beta_{2} \neq \equiv 0$, then there is a primitive element $z \in H^{2 q h}\left(X ; Z_{p}\right)$ such that $\beta_{1} z=0,\{z\} \neq 0$ in $B_{2}$, and $z^{p}=\beta_{1} w \neq 0$, where $w \in P\left(H^{*}\left(X ; Z_{p}\right)\right)$.

Proof. The element $a$ satisfies all conditions except that $b\left(\beta_{1} b=a^{p}\right)$ may not be primitive. Since $a^{p}$ is primitive, we shall find out the form of elements $c \in H^{*}\left(X ; Z_{p}\right)$ such that $\beta_{1} c$ is primitive. Since $H^{*}\left(X ; Z_{p}\right)$ is primitively generated, the differential $\beta_{1}$ is completely determined by its action on $P\left(H^{*}\left(X ; Z_{p}\right)\right)=P$, $\beta_{1}: P \rightarrow P$. Let $L=$ free associative, commutative algebra generated by $P$ over $Z_{p}$, which is made a Hopf algebra by making elements of $P$ primitive and a differential Hopf algebra by extending $\beta_{1}: P \rightarrow P$ to $d$ on all of $L$. There is a natural map of differential Hopf algebras $\eta: L \rightarrow H^{*}\left(X ; Z_{p}\right)$, induced by the inclusion of $P \rightarrow H^{*}\left(X ; Z_{p}\right)$, which is onto since $P$ generates $H^{*}\left(X ; Z_{p}\right)$.

Since $\beta_{1}: P\left(H^{2 m}\left(X ; Z_{p}\right)\right) \rightarrow P\left(H^{2 m+1}\left(X ; Z_{p}\right)\right)$ is zero [7, Theorem 6.12] (8.2), all even dimensional generators of $L$ are cycles. It is easy to verify directly that the module of primitive elements of $L$ has a basis consisting of the ring generators of $L$ (elements of $P$ ) and iterated $p$ th powers of generators. Let $x_{1}, \cdots, x_{l}$ be odd dimensional generators of $P$ such that the elements $\beta_{1} x_{1}, \cdots, \beta_{1} x_{l}$ are linearly independent, and $x_{1}, \cdots, x_{l}, \xi_{l+1}, \cdots, \xi_{q}$ are a basis for $P$ in odd dimensions where $\beta_{1} \xi_{i}=0, l+1 \leqq i \leqq q$. Then $\beta_{1} x_{1}, \cdots, \beta_{1} x_{l}$ are a linearly independent set of even dimensional elements of $P$.

Then it follows that $L \cong\left(\bigotimes_{i=1}^{l} \Lambda\left(x_{i}\right) \otimes Z_{p}\left[y_{i}\right]\right) \otimes M$, where $d x_{i}=y_{i}^{h}, h=p^{t}$, $0 \leqq t<\infty$ ( $h$ depends on $i)$, and $d(M)=0$ ( $\Lambda$ denotes exterior algebra). The isomorphism is given by inclusion on the generators $x_{i}, y_{i}$ and on $M$, where $M$ is the subalgebra of $L$ generated by $\xi_{i+1}, \cdots, \xi_{q}$ and other even dimensional elements of $P$. It follows that the isomorphism is one of coalgebras and of Hopf algebras if $p \neq 2$. It follows that if $u^{p}$ is primitive in $L$, and $u^{p}=d v$, then there is an element $u^{\prime}=d v^{\prime} \in P$ such that $\left(u-u^{\prime}\right)^{p}=d w$ and $w$ is primitive. For the linear subspace $S$ spanned by $x_{1}, \cdots, x_{l},{ }_{1} x_{1}=x_{1}\left(d x_{1}\right)^{p-1}, \cdots$, ${ }_{1} x_{l}=x_{l}\left(d x_{l}\right)^{p-1},{ }_{2} x_{1}={ }_{1} x_{1}\left(d_{1} x_{1}\right)^{p-1}, \cdots$, is such that $d(S) \supseteq P \cap($ image $d)$.

Hence, in $H^{*}\left(X ; Z_{p}\right)$ if $a^{p}=\beta_{1} b$, then there is $u^{\prime}=\beta_{1} v^{\prime} \in P$, with $z^{p}=\left(a-u^{\prime}\right)^{p}$ 
$=\beta_{1} w, w \in P$. It remains to show that $\{z\} \neq 0$ in $B_{2}$, and $z^{p} \neq 0$. But if $a-u^{\prime}=\beta_{1} x$, then $a \in$ image $\beta_{1}$, which is false as shown above. Then it follows from Corollary 4.4 that $z^{p} \neq 0$ and the lemma is proved.

Now $\beta_{1}=j_{*} \partial_{1}$, and since $w$ is primitive, i.e., if $\psi$ is the diagonal map in cohomo$\log y, \psi w=\pi_{1}^{*} w+\pi_{2}^{*} w$, it follows that $\psi\left(\partial_{1} w\right)=\pi_{1}^{*}\left(\partial_{1} w\right)+\pi_{2}^{*}\left(\partial_{1} w\right)=\partial_{1} w \otimes 1+$ $1 \otimes \partial_{1} w$. Since $z=j_{*} c, c \in H^{2 q}(X), j_{*}\left(c^{p}-\partial_{1} w\right)=0$, so that $c^{p}=\partial_{1} w+p \alpha, \alpha \in H^{*}(X)$. Since $z=j_{*} c \in P\left(H^{*}\left(X ; Z_{p}\right)\right), \psi c=c \otimes 1+1 \otimes c+p \gamma, \gamma \in H^{*}(X \times X)$.

Then

Hence

$$
\begin{aligned}
\psi c^{p} & =c^{p} \otimes 1+1 \otimes c^{p}+\sum\left(\begin{array}{l}
p \\
i
\end{array}\right) c^{p-i} \otimes c^{i}+p^{2} \delta \\
& =\partial_{1} w \otimes 1+1 \otimes \partial_{1} w+p \psi \alpha .
\end{aligned}
$$

$$
p \psi \alpha=p \alpha \otimes 1+1 \otimes p \alpha+\sum\left(\begin{array}{c}
p \\
i
\end{array}\right) c^{p-i} \otimes c^{i}+p^{2} \delta
$$

so $\psi \alpha=\alpha \otimes 1+1 \otimes \alpha+\sum b_{i} c^{p-i} \otimes c^{i}+p \delta+\eta, \quad\left(p \eta=0, \quad b_{i}=p^{-1}\left(\begin{array}{c}p \\ i\end{array}\right)\right)$. Since $\{z\} \neq 0$ in $B_{2}$, then $p c \neq 0$.

Let $\bar{z} \in H_{*}\left(X ; Z_{p}\right), z(\bar{z}) \neq 0,\{\bar{z}\} \neq 0$ in $B^{2}$. Then

$$
\begin{aligned}
\left(j_{*} \alpha\right)\left(\bar{z}^{p}\right) & =\left(\psi j_{*} \alpha\right)\left(\bar{z}^{p-1} \otimes \bar{z}\right) \\
& =\left(j_{*} \psi \alpha\right)\left(\bar{z}^{p-1} \otimes \bar{z}\right) \\
& =\left(j_{*} \alpha \otimes 1+1 \otimes j_{*} \alpha+\sum b_{i} z^{p-i} \otimes z^{i}+j_{*} \eta\right)\left(\bar{z}^{p-1} \otimes \bar{z}\right) .
\end{aligned}
$$

Since $p \eta=0, \eta=\partial_{1} \xi$ so that $j_{*} \eta=\beta_{1} \xi$. Since $\beta^{1} \bar{z}=0, \quad\left(\beta_{1} \xi\right)\left(\bar{z}^{p-1} \otimes \bar{z}\right)$ $=(\xi)\left(\beta^{1}\left(\bar{z}^{p-1} \otimes \bar{z}\right)\right)=0$. Hence for dimensional reasons

$$
\left(j_{*} \alpha\right)\left(\bar{z}^{p}\right)=\left(b_{1} z^{p-1} \otimes z\right)\left(\bar{z}^{p-1} \otimes \bar{z}\right)=b_{1}(p-1) !(z(\bar{z}))^{p} \neq 0
$$

(cf. [7, Lemma 6.2]). Then $\bar{z}^{p} \neq 0$ in $H_{*}\left(X ; Z_{p}\right)$ which contradicts the hypothesis that $H^{*}\left(X ; Z_{p}\right)$ is primitively generated. Hence $\beta_{i} \equiv 0$ so that $B_{2}=B_{\infty}$ by Corollary 4.6 and the theorem is proved.

5. Rational homology spheres which are $H$-spaces. In this section we shall study the cohomology of $\mathrm{H}$-spaces which are rational homology spheres. We shall prove a theorem (Theorem 5.3) describing the mod 2 cohomology of such $H$-spaces. We apply this theorem in $\$ 6$ and $\$ 7$, together with the results of J. F. Adams [1] to prove the following theorems (these results were announced in $[10$, Theorem 5.1] in a slightly weaker form):

THEOREM 5.1. Let $p: S^{n} \rightarrow B$ be a fibre map, with base $B$ and fibre $F a$ connected polyhedra, $B \neq$ point. Then $F$ is the homotopy type of $a 1,3$ or 7 sphere.

THEOREM 5.2. Let $X$ be a polyhedron which is an H-space and a rational 
cohomology sphere with $H_{i}(X)=0$ for large $i$. Then $X$ is the homotopy type of a 1, 3 or 7 sphere or a 3 or 7 dimensional real projective space.

The result on homology we shall need is the following:

THEOREM 5.3. Let $X$ be an $H$-space, $H_{*}(X)$ of finite type, with $H^{*}(X ; Q)=\Lambda(z)$, $\operatorname{dim} z=n$, and $H^{j}\left(X ; Z_{2}\right)=0$ for large $j$. Then $H^{*}\left(X ; Z_{2}\right)=\Lambda(x) \otimes Z_{2}[y] /\left(y^{2^{k}}\right)$ as a Hopf algebra, where $\operatorname{dim} x=2 a-1, y=S q^{1} x, n=2^{k}(2 a)-1$, provided $H^{1}\left(X ; Z_{2}\right)=0$. If $H^{1}\left(X ; Z_{2}\right) \neq 0$, then $H^{*}\left(X ; Z_{2}\right)=Z_{2}[x] / x^{2^{k+1}}, \operatorname{dim} x=1$. Note that $S q^{1} x=0$ implies $H^{*}\left(X ; Z_{2}\right)=\Lambda(x)$, which we allow as a possibility.

The proof of Theorem 5.3 proceeds by a sequence of lemmas.

The idea of the proof is to show, using the information about the Bockstein spectral sequence obtained in [9, Theorem 4.8], that the existence of more than one even dimensional generator implies the existence of $2^{r}$ torsion with $r>1$. Then the existence of such higher torsion is shown to lead to a contradiction, so that there is at most one even dimensional generator $y$, and in that case $y=S q^{1} x$.

Let $B_{k}$ denote the Bockstein spectral sequence in cohomology $\bmod 2$ of the space $X$. We first describe this spectral sequence.

LEMmA 5.4. $\quad B_{k}=\Lambda\left(\xi_{k}\right) \otimes \bigotimes_{m \geqq i \geqq q(k)} Z_{2}\left[y_{i}\right] /\left(y_{i}^{h_{i}}\right)$, where $1+\operatorname{dim} \xi_{k}=\operatorname{dim} y_{q(k)}$, $q(k)$ is an integer depending on $k, q(k) \leqq q(k+1) \leqq q(k)+1, h_{i}=2^{n_{i}}, n_{i} \geqq 1$, $h_{i} \operatorname{dim} y_{i}=\operatorname{dim} y_{i+1}$.

Proof. The case $k=1$ is simply [9, Theorem 4.8], with $\xi_{1}=x, q(1)=1$. We now proceed by induction, assuming the lemma true for $k$. We prove the following two lemmas with this hypothesis.

Lemma 5.5. For any $l, B_{k}^{l}$ has at most one nonzero element.

Proof. Since $B_{k}=\Lambda\left(\xi_{k}\right) \otimes \bigotimes_{m \geqq i \geqq q(k)} Z_{2}\left[y_{i}\right] /\left(y_{i}^{h_{i}}\right)$, any element in $B_{k}$ is a linear combination of monomials $\xi_{k}^{\varepsilon} y_{q}^{\eta_{q}} \cdots y_{m}^{\eta_{m}}, q=q(k), \varepsilon=0$ or $1,0 \leqq \eta_{j}<2^{n_{j}}$, $\operatorname{dim} y_{j+1}=2^{n_{j}} \operatorname{dim} y_{j}, 1+\operatorname{dim} \xi_{k}=\operatorname{dim} y_{q}$. Then

$$
\begin{aligned}
\operatorname{dim}\left(\xi_{k}^{\varepsilon} y_{q}^{\eta_{q}} \cdots y_{m}^{\eta_{m}}\right) & =\varepsilon \operatorname{dim} \xi_{k}+\sum_{j=q}^{m} \eta_{j} \operatorname{dim} y_{j} \\
& =\varepsilon\left(\left(\operatorname{dim} y_{q}\right)-1\right)+\sum\left(\eta_{j} \prod_{s=q}^{j-1} 2^{n_{s}}\right) \operatorname{dim} y_{q} .
\end{aligned}
$$

Clearly no two different choices of $\varepsilon, \eta_{q}, \cdots, \eta_{m}$ give the same dimension, which proves Lemma 5.5 .

LEMMA 5.6. $\beta_{k} y_{j}=0, q(k) \leqq j \leqq m$.

Proof. By Lemma 5.5, there are no decomposable elements in the dimensions of the generators $y_{j}$. Hence it follows from [9, Lemma 4.5] that $\beta_{k} y_{j}=0$. 
Now we return to the proof of Lemma 5.4. If $\beta_{k} \xi_{k}=0$, then $\beta_{k} \equiv 0$ on $B_{k}$ so that $B_{k+1}=B_{k}, \xi_{k+1}=\xi_{k}, q(k+1)=q(k)$, and Lemma 5.4 is true in this case for $k+1$. If $\beta_{k} \xi_{k} \neq 0$, then $\beta_{k} \xi_{k}=y_{q}$, and since $\beta_{k} y_{j}=0$ for all $j$, $B_{k+1}=\Lambda\left(\xi_{k+1}\right) \otimes \bigotimes_{m \geqq i \geqq q(k+1)} Z_{2}\left[y_{i}\right] /\left(y_{i}^{h_{i}}\right), h_{i}=2^{n_{i}}$, where

$$
\xi_{k+1}=\left\{\xi_{k} y_{q}^{h_{q}-1}\right\}, q=q(k), q(k+1)=q(k)+1 .
$$

This completes the proof of Lemma 5.4. Note here the possibility that $y_{1}=x^{2}=S q^{1} x$ does not affect any step in the argument, except that the proof of Lemma 5.6 becomes trivial for $y_{1}=x^{2}$.

The truth of Lemma 5.4 establishes the truth of Lemmas 5.5 and 5.6 for all $k$. From this we get:

For any abelian group $A$, let $A_{2}$ denote its 2-primary component.

LEMMA 5.7. For $1 \leqq i \leqq m$, there are elements $w_{i} \in H^{*}(X ; Z)_{2}$ such that $j_{*}\left(w_{i}\right)=y_{i} \in H^{*}\left(X ; Z_{2}\right)\left(j: Z \rightarrow Z_{2}\right.$ is reduction $\left.\bmod 2\right)$.

Proof. It follows from Lemma 5.6 that the $\left\{y_{i}\right\}$ are permanent cocycles in the Bockstein spectral sequence, from which it follows that they are in the image of $j_{*}$ (see [7, Theorem 3.11]), so in particular in $j_{*}\left(H^{*}(X ; Z)_{2}\right)$.

\section{LEMMA 5.8. Order $w_{i+1}>\operatorname{order} w_{i}$.}

Proof. Since $j_{*}\left(w_{i+1}\right)=y_{i+1}, j_{*}\left(w_{i}\right)=y_{i}$, and since by Lemma $5.4, y_{i+1}$ persists longer in the spectral sequence than $y_{i}$ (i.e., if $q(k)=i$, and $y_{i}=0$ in $B_{k+1}$, then $q(k+1)=i+1$, so that $y_{i+1} \neq 0$ in $\left.B_{k+1}\right)$, it follows that order $w_{i+1}>$ order $w_{i}$.

LEMMA 5.9. Let $z=w_{i}^{k}$, where $k=2^{n_{i}-1}, 1 \leqq i \leqq m$, and suppose $2 z \neq 0$. Then $y_{i+1}=j_{*}\left(w_{i+1}\right) \neq 0$, i.e., $m>i$.

Proof. Let $u=j_{*}(z)=y_{i}^{k}$. Let $\mu: X \times X \rightarrow X$ be the multiplication on $X$. Denote by $A_{i}$ the subalgebra of $H^{*}\left(X ; Z_{2}\right)$ generated by $x, y_{1}, \cdots, y_{i-1}$. Then $A_{i}^{j}=0$ in dimensions $j \geqq a_{i}\left(a_{i}=\operatorname{dim} y_{i}\right)$.

Now $\mu^{*}\left(y_{i}\right)-y_{i} \otimes 1-1 \otimes y_{i} \in A_{i} \otimes A_{i}$, for dimensional reasons. It follows that $\left(\mu^{*}\left(y_{i}\right)-y_{i} \otimes 1-1 \otimes y_{i}\right)^{2}=0$, i.e., $y_{i}^{2}$ is primitive, since this element is in $\sum_{q+l=2 a_{i}} A_{i}^{l} \otimes A_{i}^{q}$, and either $l$ or $q \geqq a_{i}$.

Now $\operatorname{dim} z=k a_{i}$. It suffices to show that $H^{2 k a_{i}}\left(X ; Z_{2}\right) \neq 0$. For no decomposable element may have dimension $2 k a_{i}=2^{n i} a_{i}$, so if $H^{2 k a_{i}}\left(X ; Z_{2}\right) \neq 0$, there must be a new generator

$$
y_{i+1}=j_{*}\left(w_{i+1}\right) \in H^{2 k a_{i}}\left(X ; Z_{2}\right) .
$$

Now, if $k>1\left(n_{i}>0\right)$, then $y_{i}^{2}$ is primitive, so $u=y_{i}^{k}$ is primitive. Since $u=j_{*}(z)$ and $2 z \neq 0$, then it follows that $z$ satisfies the hypothesis of Theorem 4.3, with $r=2, s=1$, and hence $u$ has 1 -implication in $H^{*}\left(X ; Z_{2}\right)$ so that $H^{2 k a_{i}}\left(X ; Z_{2}\right) \neq 0$. 
If $k=1$, then $z=w_{i}$, and $u=y_{i}$ is indecomposable in $H^{a_{i}}\left(X ; Z_{2}\right)$. Hence, there is a homology class $\bar{y}_{i}$, dual to $y_{i}$ (i.e., $y_{i}\left(\bar{y}_{i}\right) \neq 0$ ), with $\bar{y}_{i}$ primitive in $H_{a_{i}}\left(X ; Z_{2}\right)$, and $\beta_{1} \bar{y}_{i}=0$ (since $2 z=0$ ). Hence $\bar{y}_{i}$ has 1 -implication by Corollary 4.2 (note that $a_{i}$ is even), so that $H_{2 a_{i}}\left(X ; Z_{2}\right) \neq 0$ and thus $H^{2 a_{i}}\left(X ; Z_{2}\right) \neq 0$, which completes the proof of Lemma 5.9.

Proof of Theorem 5.3. By Lemma 5.4 with $k=1$ (see [9, Theorem 4.8]),

$$
H^{*}\left(X ; Z_{2}\right)=\Lambda(x) \otimes \bigotimes_{1 \leqq i \leqq m} Z_{2}\left[y_{i}\right] /\left(y_{i}^{h_{i}}\right) .
$$

By Lemma 5.7, $y_{i}=j_{*}\left(w_{i}\right), w_{i} \in H^{*}\left(X ; Z_{2}\right)$, and by Lemma 5.8, order $w_{i+1}>$ order $w_{i}$. Hence $2 w_{i} \neq 0$, for $i>1$. It follows from Lemma 5.4 that if $k=2^{n_{i}-1}, w_{i}^{k}$ has the same order as $w_{i}$, so $2 w_{i}^{k} \neq 0$ for $i>1$. Then Lemma 5.9 implies $w_{i+1} \neq 0$ if $w_{i} \neq 0$ for $i>1$. But applying this reasoning to $w_{m}$, the highest dimensional $w_{i}$, we get a contradiction unless $m=1$. Similarly, if $y_{1} \neq 0$ and $S q^{1} x=0$, then $2 w_{1} \neq 0$ (i.e., $w_{1}$ is not of order 2 ), so that $w_{2} \neq 0$ by Lemma 5.9 , which is again a contradiction. Hence either $S q^{1} x=y_{1}$ and

$$
H^{*}\left(X ; Z_{2}\right)=\Lambda(x) \otimes Z_{2}\left[S q^{1} x\right] /\left(S q^{1} x\right)^{h}, \text { or } y_{1}=0 \text { and } H^{*}\left(X ; Z_{2}\right)=\Lambda(x),
$$

which completes the proof of Theorem 5.3.

6. Fiberings of spheres. In this section we prove Theorem 5.1.

Let $A$ be a graded algebra over a field $K$. Then the set $\left\{x_{1}, \cdots, x_{m}\right\}, x_{i} \in A$, is called a simple system of generators for $A$ if the monomials $x_{i_{1}} \cdots x_{i_{k}}$, $1 \leqq i_{1}<i_{2}<\cdots<i_{k} \leqq m$, form an additive basis for $A$ over $K$, where $\operatorname{dim} x_{i}$ is assumed odd for all $i$ if characteristic $K \neq 2$.

We recall a theorem of Borel:

THEOREM (BoreL). Let $p: E \rightarrow B$ be a fibre map, with fibre $F$. Let $K$ be a field, suppose $H^{*}(F ; K)$ has a simple system of transgressive generators $\left\{x_{1}, \cdots, x_{m}\right\}$, and suppose $H^{t}(E ; K)=0$ for $t<n$. Then $H^{*}(B ; K) \cong K\left[y_{1}, \cdots, y_{m}\right]$ in dimensions $<n$, where $y_{i}$ is a transgression image of $x_{i}$ for each $i$.

This theorem is [5, Theorem 13.1(b)] and [5, Proposition 16.1]. It follows easily by a standard argument from a comparison theorem, such as $[15$, Proposition 5.3].

THEOREM 6.1. Let $p: E \rightarrow B$ be a fibre map, with connected fibre $F$, $H^{i}(B ; K)=H^{i}(F ; K)=0$ for large $i, K$ a field. Suppose that $H^{*}(F ; K)$ has a simple system of transgressive generators and that $H^{*}(E ; K)=\Lambda(\zeta), \operatorname{dim} \zeta=n$. Then $H^{*}(F ; K)=\Lambda(x)$, i.e., the simple system of generators has only one element.

Proof. Let $E_{r}=$ cohomology spectral sequence for $p$ over $K$. Let $x_{1}, \cdots, x_{m}$ be a simple system of transgressive generators for $H^{*}(F ; K)$. (Note that if characteristic of $K \neq 2$, then $\operatorname{dim} x_{i}$ is odd for each $i$.) Then $x_{1} x_{2} \cdots x_{m}$ is the unique element of highest dimension in $H^{*}(F ; K)$. Let $H^{i}(B ; K)=0$ for $i>q, H^{q}(B ; K) \neq 0$. 
If $u \neq 0 \in H^{q}(B ; K)$, then the total degree of $z=x_{1} \cdots x_{m} \otimes u \geqq$ total degree $w$ for any $w \in E_{2}$, so $z$ is a permanent cocycle in $E_{r}$. Further, the fibre degree of $z \geqq$ fibre degree of $w$, for any $w \in E_{2}$, and since $d_{r}$ lowers fibre degree for $r \geqq 2, z$ is not a coboundary in any $E_{r}$. Hence $\{z\} \neq 0 \in E_{\infty}$ and, since $E_{\infty} \cong H^{*}(E ; K)$, it follows that $\operatorname{dim} z=n$.

Assume $\operatorname{dim} x_{i} \leqq \operatorname{dim} x_{i+1}$, for each $i$. Now $x_{i}$ transgresses, so that if $k=\operatorname{dim} x_{i}+1$, then there is an element $y_{i} \in E_{2}^{k, 0}$, with $d_{k} x_{i}=\left\{y_{i}\right\}$. By the theorem of Borel, $H^{*}(B ; K)=K\left[y_{1}, \cdots, y_{m}\right]$ in dimensions $<n$. Hence, if $\operatorname{dim} y_{1}=k, y_{1}^{l} \neq 0$ for $l<n / k$, and since $H^{i}(B ; K)=0$ for $i>q$, and $n>q, y_{1}^{l}=0$ for $l \geqq n / k$. Let $s=$ greatest integer $<n / k$. Then $x_{1} \otimes y_{1}^{s} \neq 0$ in $E_{2}$ and $d_{k}\left(x_{1} \otimes y_{1}^{s}\right)=y_{1}^{s+1}=0$. Hence, since fibre degree $x_{1} \otimes y_{1}^{s}=k-1, x_{1} \otimes y_{1}^{s}$ is a permanent cocycle in $E_{r}$. Now $t=\operatorname{dim}\left(x_{1} \otimes y_{1}^{s}\right)=k-1+s k<n$, if $m>1$. In dimensions $\leqq t<n$, the boundary in the spectral sequence is completely determined by its action on the $x_{i}$ 's, and the product structure, which is simply

$$
\Lambda\left(x_{1}, \cdots, x_{m}\right) \otimes K\left[y_{1}, \cdots, y_{m}\right]
$$

in these dimensions if characteristic of $K \neq 2$, and yields the same result even when characteristic $K=2$, since $x_{1}, \cdots, x_{m}$ is a simple system of generators. Hence $\left\{x_{1} \otimes y_{1}^{s}\right\}$ cannot be a boundary and its class is nonzero in $E_{\infty}$. Then since $E_{\infty} \cong \Lambda(z), x_{1} \otimes y_{1}^{s}=z, m=1$ and Theorem 6.1 is proved.

Now let $p: S^{n} \rightarrow B$ be a fibre map, $B$ connected. Then $p$ is onto so that $B$ is a compact polyhedron, and $H^{i}(B)=0$ for large $i$. The fibre $F$ is a closed subset of $S^{n}$ so that $F$ is a compact polyhedron, and $H^{i}(F)=0$ for large $i$.

If $B$ is not a single point then the fibre $F$ is not all of $S^{n}$. It follows that $F$ is contractible in $S^{n}$, so that by a theorem of E. H. Spanier and J. H. C. Whitehead [19], $F$ is an $H$-space. (This result is deduced from the covering homotopy theorem.) Then by Hopf's Theorem [5, Proposition 7.2] (or [16, Theorem 4.6.]) on $H$-spaces, if $Q=$ rational numbers and $F$ is connected, then $H^{*}(F ; Q)=\Lambda\left(x_{1}, \cdots, x_{m}\right)$ where $\operatorname{dim} x_{i}$ is odd, $1 \leqq i \leqq m$. By Borel's transgression theorem [5, Theorem 13.1(a)], the $x_{i}$ may be chosen transgressive so that $\left\{x_{1}, \cdots, x_{m}\right\}$ are a simple system of transgressive generators for $H^{*}(F ; Q)$. Hence by Theorem 6.1 , it follows that $m=1$, and we get the result of Borel [4] that $F$ is a rational homology sphere.

We now apply Theorem 5.3, and we get that $H^{*}\left(F ; Z_{2}\right)=\Lambda(x) \otimes Z_{2}[y] /\left(y^{h}\right)$, where $y=S q^{1} x, h=2^{n}$ and $y=x^{2}$ if $\operatorname{dim} x=1\left(y=S q^{1} x=0\right.$ being allowed). Now $x$ is transgressive since it is the lowest dimensional element in the fibre, and a Steenrod operation on a transgressive element yields a transgressive element. Hence $\left\{x, y, y^{2}, y^{4}, \cdots, y^{h / 2}\right\}$ is a simple system of transgressive generators for $H^{*}\left(F ; Z_{2}\right)$ (note that $z^{2}=S q^{l} z$, if $\operatorname{dim} z=l$ ). Therefore, by Theorem 6.1, $H^{*}\left(F ; Z_{2}\right)=\Lambda(x)$, i.e., $S q^{1} x=0$.

J. F. Adams [1] has shown that if a mod 2 homology $n$-sphere is an $H$-space, 
then $n=1,3$, or 7 . Then $F$ is a rational homology 1,3 or 7 sphere (this follows easily using the Bockstein spectral sequence $\bmod 2$, since $E_{1}=E_{\infty}$ ). By [9, Theorem 4.8$]$, if $H^{*}(F ; Z)$ has $p$-torsion for a prime $p$, then $n=2 q p^{\alpha}-1$, for some $q \geqq 1, \alpha \geqq 1$. It follows easily that $H^{*}(F ; Z)$ has no $p$-torsion for any odd prime $p$, since $n=1,3$ or 7 . Hence $F$ is a homology 1,3 or 7 sphere.

Since $F$ is an $H$-space, $\pi_{1}(F)$ is abelian, so $\pi_{1}(F)=H_{1}(F ; Z)$. If $n=3$ or 7 , it follows that $F$ is simply connected. Then one can easily deduce that $F$ is homotopy equivalent to $S^{3}$ or $S^{7}$, since there is a map of $S^{3}$ or $S^{7}$ into $F$ which induces isomorphism on homology groups (using the Hurewicz and Whitehead theorems). If $n=1$, then we have a map $f: S^{1} \rightarrow F$ which induces an isomorphism of homology, but since the spaces are not simply connected we cannot immediately apply Whitehead's theorem.

However, the result follows in this case from the following:

Lemma 6.2. Let $X$ and $Y$ be connected $H$-spaces $\left({ }^{5}\right), f: X \rightarrow Y$, a map inducing isomorphism of homology. Then the map of universal covering spaces $f^{\prime}: X \rightarrow \bar{Y}$ also induces isomorphism of homology $f^{\prime}: H_{*}(\bar{X}) \cong H_{*}(\bar{Y})$, so that $f^{\prime}$ and $f$ are homotopy equivalences if $X$ and $Y$ are polyhedra.

Proof. Let $E=$ the space of paths in $X$ which start at the identity of $X$, i.e., if $\alpha \in E, \alpha: I \rightarrow X, \alpha(0)=e$. Then $E$ is an $H$-space, with multiplication pointwise of paths as the product, i.e., $(\alpha \cdot \beta)(t)=\alpha(t) \cdot \beta(t)$ (product in $X$ ). Then the projection $\pi: E \rightarrow X,(\pi(\alpha)=\alpha(1))$, is a homomorphism of $H$-spaces. Now $\bar{X}$ is the set of equivalence classes of $E$, where $\alpha \sim \beta$ if $\alpha(1)=\beta(1)$ and $\alpha$ is homotopic to $\beta$ rel 0 and 1 . It follows easily that $\bar{X}$ is an $H$-space, the projection $\bar{\pi}: \bar{X} \rightarrow X$ is a homomorphism of $H$-spaces, $\pi_{1}(X)=\bar{\pi}^{-1}(e)$, and the action of $\alpha \in \pi_{1}(X)$ on $X$ by "deck-translation" is the same as the action of $\alpha \in \pi_{1}(X) \subseteq X$ acting by the left multiplication of $X$. Since $X$ is connected, left multiplication by an element of $X$ is homotopic to the identity map of $X$, so that $\pi_{1}(X)$ acts trivially on $H_{*}(\bar{X})$.

By replacing the spaces involved by others of the same homotopy type, we may consider $X$ as a fibre space over $K(\pi, 1)$ with fibre $\bar{X}$, where $\pi=\pi_{1}(X)$, and similarly with $Y$ (see [18] or [5]). Then $f$ induces a map of fibre spaces

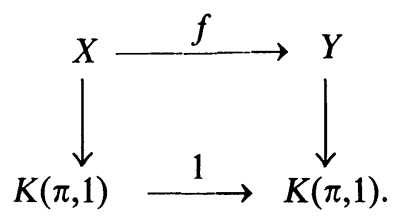

The systems of local coefficients $H_{*}(\bar{X})$ and $H_{*}(\bar{Y})$ are trivial by the above re-

(5) One may replace this hypothesis by the hypothesis that $\pi_{1}(X), \pi_{1}(Y)$ act trivially on $H_{*}(\bar{X}), H_{*}(\bar{Y})$, respectively, and assume also that $f_{*}: \pi_{1}(X) \rightarrow \pi_{1}(Y)$ is an isomorphism. It is in fact the first part of the proof of the lemma to prove this condition. 
marks, and since $f_{*}: H_{*}(X) \cong H_{*}(Y)$ and the map on the base spaces $K(\pi, 1)$ is the identity, by the Comparison Theorem (see [15, Proposition 5.3] for example), $f_{*}^{\prime}: H_{*}(\bar{X}) \rightarrow H_{*}(\bar{Y})$ is an isomorphism, and thus by the theorem of J. H. C. Whitehead, $f^{\prime}$ and $f$ are homotopy equivalences.

This completes the proof of Theorem 5.1.

7. Homotopy type of $H$-spaces which are rational homology spheres. In this section we prove Theorem 5.2. We first use the device of the "projective plane of an $H$-space $X$ "' together with Theorem 5.3 to show that the homology of $X$ is the same as that of one of the spaces $S^{1}, S^{3}, S^{7}, P^{3}$ or $P^{7}$. Then we shall show that a map can be constructed inducing the homology isomorphism, so that applying Lemma 6.2 , the result follows.

The projective plane $P_{2} X$ of an $H$-space $X$ is described in [20], and various cohomology properties of it are obtained in [12].

Let $X$ be a connected polyhedron which is an $H$-space, with $H_{i}\left(X ; Z_{2}\right)=0$ for large $i$, and a rational homology sphere. Then by Theorem 5.3, $H^{*}\left(X ; Z_{2}\right)=\Lambda(x) \otimes Z_{2}[y] /\left(y^{h}\right)$ where $y=S q^{1} x, h=2^{n}$. If $S q^{1} x=0$, then as we saw in $\S 6, X \cong S^{1}, S^{3}$ or $S^{7}$. Let us assume $S q^{1} x=y \neq 0$. Now $x$ is primitive, since it is in lowest nonzero dimension of $H^{*}\left(X ; Z_{2}\right)$, therefore $S q^{1} x=y$ is primitive, and $H^{*}\left(X ; Z_{2}\right)$ is primitively generated. It follows from [12, Theorem 1.1], if $\operatorname{dim} y=2 n$ and $n>1$, that $H^{*}\left(P_{2} X ; Z_{2}\right)$ has as a basis over $Z_{2}$, in dimensions $\leqq 4 n+2, \xi, \eta, \xi^{2}, \xi \eta, \zeta, \eta^{2}$, where $\operatorname{dim} \xi=2 n, \iota(\xi)=x, \iota(\zeta)=y^{2}, \eta=S q^{1} \xi$; $\iota$ is a map defined in [12], $\iota: H^{k}\left(P_{2} X\right) \rightarrow H^{k-1}(X)$. Then $H^{j}\left(P_{2} X ; Z_{2}\right)=0$, $2 n+1<j<4 n$. Since $\eta^{2}=S q^{2 n+1} \eta \neq 0$ by [12, Theorem 1.1], we have $S q^{1} S q^{2 n} S q^{1} \xi=\eta^{2} \neq 0$ in $H^{*}\left(P_{2} X ; Z_{2}\right)$.

Now $n$ must be even. For if $n$ is odd, then $S q^{2 n}$ is factorizable in $S q^{i}$ 's for $i<2 n$. Since $H^{j}\left(X ; Z_{2}\right)=0$ for $2 n+1<j<4 n, S q^{2 n} \xi=\gamma\left(S q^{1} \xi\right)$ where $\gamma$ is an element of degree $2 n-1$ in the Steenrod algebra. But since $\gamma$ is of odd degree, it must be factorizable, which is impossible. Hence $n$ is even. (This argument could be extended to show that $n=2^{q}$.)

Then consider the Adem relation (see [2])

$$
S q^{n} S q^{n+1}=\sum_{0 \leqq t \leqq n / 2}\left(\begin{array}{c}
n-t \\
t
\end{array}\right) S q^{n+1-t} S q^{t}
$$

Since $n$ is even, we get

$$
S q^{n} S q^{n+1}=S q^{2 n+1}+S q^{2 n} S q^{1}+\sum_{2 \leqq t \leqq n / 2} a_{t} S q^{2 n+1-t} S q^{t}, a_{t} \in Z_{2}
$$

If we apply both sides to $\xi$, since $\xi \in H^{2 n}\left(P_{2} X ; Z_{2}\right)$, and $H^{j}\left(P_{2} X ; Z_{2}\right)=0$ for $2 n+1<j<4 n, S q^{t} \xi=0$ for $2 \leqq t \leqq n / 2, S q^{2 n+1} \xi=0$, and $S q^{n+1} \xi=0$, if $n>1$. Hence if $n>1 S q^{2 n} S q^{1} \xi=0$, and thus $\eta^{2}=S q^{1} S q^{2 n} S q^{1} \xi=0$ which is a contradiction. Hence, if $S q^{1} x \neq 0$ in $H^{*}\left(X ; Z_{2}\right)$, then $n=1$. 
THEOREM 7.1. Let $X$ be a connected polyhedron which is an H-space, $H_{i}\left(X ; Z_{2}\right)=0$ for large $i$, and $H^{*}(X ; Q)=\Lambda(z)$, i.e., $X$ is a rational homology sphere. Then $H^{*}\left(X ; Z_{2}\right)=\Lambda(x), \operatorname{dim} x=1,3$ or 7 or $H^{*}\left(X ; Z_{2}\right)=Z_{2}[x] /\left(x^{h}\right)$, $h=4$ or $8, \operatorname{dim} x=1$.

Proof. Let us suppose $S q^{1} x \neq 0$, so that $H^{*}\left(X ; Z_{2}\right)=Z_{2}[x] /\left(x^{h}\right), h=2^{q}$, $q>1, \operatorname{dim} x=1$, by the above argument. It remains to show that $q=2$ or 3 . If we compute $H^{*}\left(\bar{X} ; Z_{2}\right)$, where $\bar{X}=$ universal covering space of $X$, using the results of [6], we get $H^{*}\left(\bar{X} ; Z_{2}\right)=\Lambda(z), \operatorname{dim} z=2^{q}-1$. Since $\bar{X}$ is an $H$-space, it follows from Adams [1] that $q=2$ or 3. This proves Theorem 7.1.

In particular, if $H_{i}(X)=0$ for large $i$, it follows that $H_{*}(X)$ has no odd torsion, as in $\S 6$, and that $\bar{X}$ is homotopy equivalent to $S^{3}$ or $S^{7}$.

Then Theorem 5.2 will follow from:

THEOREM 7.2. Let $X$ be a connected polyhedron which is an H-space with $H_{i}(X)=0$ for large $i, H^{*}\left(X ; Z_{2}\right)=Z_{2}[x] /\left(x^{h}\right), h=4$ or $8, \operatorname{dim} x=1$. Then $X$ is homotopy equivalent to the real projective space $P^{h-1}$.

Proof. As in the proof of Lemma 6.2, we consider $X$ as a fibre space over $K\left(Z_{2}, 1\right)$ with fibre $\bar{X}$. There is a natural map $g$ of $P^{h-1}$ into $K\left(Z_{2}, 1\right)$, which induces an isomorphism of $\pi_{1}\left(P^{h-1}\right)$ with $Z_{2}$. We would like to lift this map to a map $f: P^{h-1} \rightarrow X$, such that $\pi \circ f=g\left(\pi: X \rightarrow K\left(Z_{2}, 1\right)\right)$. The first obstruction to lifting $g$ is $g^{*}(k)$, where $k \in H^{h}\left(K\left(Z_{2}, 1\right) ; Z\right)$ is the first $k$-invariant of $X$. Since $X$ is an $H$-space, the $k$-invariant is in an ordinary cohomology group (without local coefficients) and it is easy to see that $k=z^{h / 2}$, where $z$ is a generator of $H^{2}\left(K\left(Z_{2}, 1\right) ; Z\right)$. Since $H^{h}\left(P^{h-1} ; Z\right)=0$, the first obstruction to lifting $g$ is zero. But the higher obstructions are in still higher cohomology groups so that all obstructions are zero and there exists a map $f: P^{h-1} \rightarrow X$, with $\pi \circ f=g$.

It is obvious that $f_{*}$ is an isomorphism of $H_{*}\left(P^{h-1} ; Z_{2}\right)$ with $H_{*}\left(X ; Z_{2}\right)$, since it is an isomorphism on cohomology $\bmod 2$, both cohomology rings being $Z_{2}[x] /\left(x^{h}\right)$ and the generator being sent to the generator. However, for any given $f, f_{*}$ may not be an isomorphism on integral homology. We shall show that $f$ can be changed so that it is an isomorphism on integral cohomology, and hence on integral homology.

Let us consider the Moore-Postnikov system of the fibre map $\pi: X \rightarrow K\left(Z_{2}, 1\right)$ (see [17]).

The first term is a fibre space $\pi^{(1)}: X^{(1)} \rightarrow K\left(Z_{2}, 1\right)$, with fibre $K(Z, h-1)$ (which is the first term of the Postnikov system of $\bar{X}$ ). There is a commutative diagram

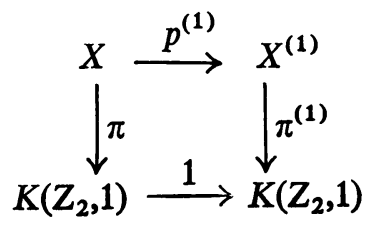


where $p^{(1)}$ is a homotopy equivalence through dimension $h-1$, i.e., $p_{*}^{(1)}$ is an isomorphism of $\pi_{i}(X)$ with $\pi_{i}\left(X^{(1)}\right)$ for $0 \leqq i \leqq h-1$. Then $f^{(1)}=p^{(1)} \circ f: P^{h-1} \rightarrow X^{(1)}$, with $\pi^{(1)} \circ f^{(1)}=g$. Since dimension $P^{h-1}=h-1$, if we can modify $f^{(1)}$ to make it an isomorphism on cohomology through dimension $h-1$, then the modified map can be factored through $X$ and induces isomorphism with the cohomology of $X$.

Now $\pi^{(1)}$ is a principal fibre space, with group $K(Z, h-1)$, so that there is an action $\mu: K(Z, h-1) \times X^{(1)} \rightarrow X^{(1)}$, which commutes with the projection $\pi^{(1)}$. Given a map $\gamma: P^{h-1} \rightarrow K(Z, h-1)$, we may modify $f^{(1)}$ to a new map $l=\mu \circ\left(\gamma, f^{(1)}\right) \circ \Delta$, and $\pi^{(1)} \circ l=g$ (here $\Delta$ is the diagonal map $P^{h-1} \rightarrow P^{h-1} \times P^{h-1}$ ). Then $l^{*}=\Delta^{*} \circ\left(\gamma^{*}, f^{(1) *}\right) \circ \mu^{*}$. Let $\xi$ be a generator of $H^{h-1}\left(X^{(1)} ; Z\right)$. From dimensional reasons and naturality of $\mu$, it follows that $\mu^{*}(\xi)=i^{*}(\xi) \otimes 1+1 \otimes \xi$, where $i: K(Z, h-1) \rightarrow X^{(1)}$ is the inclusion of the fibre. By a spectral sequence argument, it follows that $i^{*}(\xi)=2 \iota, \iota=$ fundamental class of $H^{h-1}(K(Z, h-1) ; Z)$. Then $l^{*}(\xi)=\Delta^{*}\left(2 \gamma^{*}(\iota) \otimes 1+1 \otimes f^{(1) *}(\xi)\right)=2 \gamma^{*}(\iota)+f^{(1) *}(\xi)$.

If we could choose $l$ so that $l^{*}(\xi)=x$ the generator of $H^{h-1}\left(P^{h-1} ; Z\right)$, then $l$ would be an isomorphism on all integral cohomology. Since $l^{*}=f^{(1) *}$ on $H^{*}\left(X ; Z_{2}\right)$, it follows that $f^{(1) *}(\xi)=x+2 \eta$. Hence if we choose $\gamma$ so that $\gamma^{*}(\iota)=-\eta$, then the modified map $l^{*}$ will send $\xi$ to $x$, and we are done.

8. A summary of "implication" theorems. In this section we present the principal "implication" theorems about the homology of $H$-spaces, from various papers $\left({ }^{6}\right)$.

Let $X$ be a connected $H$-space with $H_{*}(X)$ of finite type, throughout this section. (Some of these theorems are true in a slightly wider context as noted in the introduction.) $B^{r}$ and $B_{r}$ will denote the homology and cohomology Bockstein spectral sequences of $X, \bmod p$.

(8.1) Let $x \in P\left(B_{2 m}^{r}\right), x=\beta^{r} y$. Then $x$ has $\infty$-implications [7, Theorem 6.1].

(8.2) Let $\xi \in P\left(B_{r}^{2 m}\right), \beta_{r} \xi \neq 0$. Then $\xi$ has $\infty$-implications [7, Theorem 6.12].

(8.3) Let $X$ be homotopy commutative and let ${ }_{2} B_{r}$ be the cohomology Bockstein spectral sequence $\bmod 2$. If $\xi \in P\left({ }_{2} B_{r}^{2 m}\right)$, then $\xi$ has $\infty$-implications [8, Theorem 8.1].

(8.4) Let $X$ be homotopy associative and homotopy commutative. If $\xi \in P\left(B_{r}^{2 m}\right)$, then $\xi$ has $\infty$-implications (no restriction on $p$ ) [8, Theorem 8.2].

(8.5) Let $H_{*}\left(X ; Z_{p^{q}}\right)$ be associative and commutative for all $q$. If $\xi \in P\left(B_{r}^{2 m}\right)$, $r>1$, then $\xi$ has $\infty$-implications [11, Theorem 2.3].

(8.6) Let $x \in P\left(H_{2 m}\left(X ; Z_{p}\right)\right)$, with $\beta_{1} x=0$. Then $x$ has 1-implication (Corollary 4.2).

(8.7) Let $\xi \in P\left(B_{s}^{2 m}\right), \beta_{j} \xi=0$ for $s \leqq j \leqq r$ and $\{\xi\} \neq 0$ in $B_{r}^{2 m}, r>s$. Then $\xi$ has $(r-s)$-implications (in $B_{s}$ ) (Corollary 4.4).

(6) I would like to take this opportunity to acknowledge that my proof of the implication theorem announced in Abstract 553-79, Notices Amer. Math. Soc. 5 (1958), 820, was incorrect. I do not know whether the theorem is true or not as stated. 
(8.8) Suppose $\beta_{m} \equiv 0$, for some $m$. If $\xi \in P\left(B_{s}^{2 m}\right), \beta_{J} \xi=0$ for $s \leqq j \leqq r$, $s \leqq m \leqq r,\{\xi\} \neq 0$ in $B_{r}^{2 m}$, then $\xi$ has $\infty$-implications (in $B_{s}$ ) (Theorem 4.5).

(8.9) Let $\xi \in P\left(B_{\infty}^{2 m}\right)$. Then $\xi$ has $\infty$-implications (Theorem 4.7).

This is not a complete list of "implication" theorems in these papers; for instance, in [8] there are various theorems with hypothesis involving a map into an $\mathrm{H}$-space. Also we have presented the theorems in this list in the same format, with reference only to the Bockstein spectral sequence. In the papers in which they occur, they are usually given also in other forms. Note that in theorems in which only one term of the Bockstein spectral sequence is mentioned, " $n$-implications" means in that term.

\section{BIBLIOGRAPHY}

1. J. F. Adams, On the non-existence of elements of Hopf invariant one, Ann. of Math. (2) 72 (1960), 20-104.

2. J. Adem, The iteration of the Steenrod squares in algebraic topology, Proc. Nat. Acad. Sci. U.S.A. 38 (1952), 720-726.

3. - Relations on iteratedreduced powers, Proc. Nat. Acad. Sci. U.S.A.39(1953),636-638.

4. A. Borel, Impossibilité de fibre une sphère par un produit de sphères, C. R. Acad. Sci. Paris 231 (1950), 943-945.

5. - - Sur la cohomologie des espaces fibrés principaux et des espaces homogènes de groupes de Lie compacts, Ann. of Math. (2) 57 (1953), 115-207.

6. W. Browder, The cohomology of covering spaces of $H$-spaces, Bull. Amer. Math. Soc. 65 (1959), 140-141.

7. - Torsion in H-spaces, Ann. of Math. (2) 74 (1961), 24-51.

8. - Homotopy commutative H-spaces, Ann. of Math. (2) 75 (1962), 283-311.

9. _- On differential Hopf algebras, Trans. Amer. Math. Soc. 107 (1963), 153-176.

10. - Fiberings of spheres and H-spaces which are rational homology spheres, Bull. Amer. Math. Soc. 68 (1962), 202-203.

11. W. Browder and I. Namioka, H-spaces with commutative homology rings, Ann. of Math. 75 (1962), (2) 449-451.

12. W. Browder and E. Thomas, On the projective plane of an $\mathrm{H}$-space, Illinois J. Math. 7 (1963), 492-502.

13. J. Eells and N. H. Kuiper, Closed manifolds which admit non- degenerate functions with three critical points, Indag. Math. 23 (1961), 411-417.

14. S. Eilenberg and N. E. Steenrod, Foundations of algebraic topology, Princeton Univ. Press, Princeton, N.J., 1953.

15. T. Kudo and S. Araki, Topology of $H_{n}$-spaces and $H$-squaring operations, Mem. Fac. SciKyūshü Univ. Ser. A 10 (1956), 85-120.

16. J. W. Milnor and J. C. Moore, On the structure of Hopf algebras, mimeographed, Princeton, 1958.

17. J. C. Moore, Semi-simplicial complexes and Postnikov systems, Symposium Internacional de Topologia Algebraica, pp. 232-247, Univ. Nac. Autonoma de Mexico. Mexico, D.F., 1958.

18. J.-P. Serre, Groupes d'homotopie et classes de groupes abéliens, Ann. of Math. (2) 58 (1953), 258-294.

19. E. H. Spanier and J. H. C. Whitehead, On fibre spaces in which the fibre is contractible, Comment. Math. Helv. 29 (1955), 1-8.

20. J. Stasheff, Onhomotopy Abelian H-spaces, Proc. Cambridge Philos. Soc. 57(1961), 734-745.

CORNELL UNIVERSITY, ITHACA, NEW YORK 